\title{
Vibration and Sound Transmission Performance of Sandwich Panels with Uniform and Gradient Auxetic Double Arrowhead Honeycomb Cores
}

\author{
Qing $\mathrm{Li}^{1,2,3}$ and Deqing Yang $\mathbb{D}^{1,2,3}$ \\ ${ }^{1}$ State Key Laboratory of Ocean Engineering, Shanghai Jiao Tong University, Shanghai 200240, China \\ ${ }^{2}$ Collaborative Innovation Center for Advanced Ship and Deep-Sea Exploration, Shanghai Jiao Tong University, \\ Shanghai 200240, China \\ ${ }^{3}$ School of Naval Architecture Ocean and Civil Engineering, Shanghai Jiao Tong University, Shanghai 200240, China \\ Correspondence should be addressed to Deqing Yang; yangdq@sjtu.edu.cn
}

Received 19 December 2018; Accepted 21 March 2019; Published 16 April 2019

Academic Editor: Rui Moreira

Copyright (c) 2019 Qing Li and Deqing Yang. This is an open access article distributed under the Creative Commons Attribution License, which permits unrestricted use, distribution, and reproduction in any medium, provided the original work is properly cited.

\begin{abstract}
Auxetic mechanical metamaterials that exhibit a negative Poisson's ratio (NPR) can be artificially designed to exhibit a unique range of physical and mechanical properties. Novel sandwich structures composed of uniform and gradient auxetic double arrowhead honeycomb (DAH) cores were investigated in terms of their vibration and sound transmission performance stimulated by nonhomogeneous metamaterials with nonperiodic cell geometries. The spectral element method (SEM) was employed to accurately evaluate the natural frequencies and dynamic responses with a limited number of elements at high frequencies. The results indicated that the vibrating mode shapes and deformations of the DAH sandwich models were strongly affected by the patterned gradient metamaterials. In addition, the sound insulation performance of the considered DAH sandwich models was investigated regarding the sound transmission loss (STL) from $1 \mathrm{~Hz}$ to $1500 \mathrm{~Hz}$ under a normal incident planar wave, and this performance was compared with that for hexagonal honeycomb sandwich panels. A programmable structural-acoustic optimization was implemented to maximize the STL while maintaining a constant weight and high strength. The results showed that the uniform DAH sandwich models with larger NPRs generally exhibited better vibration and acoustic attenuation behaviors and that the optimized gradient increasing NPR models yielded higher STL values than the optimized gradient decreasing NPR models for two specified frequency cases, with improvements of $6.52 \mathrm{~dB}$ and $2.52 \mathrm{~dB}$ and a higher bending stiffness but a lower overall STL. Thus, sandwich panels consisting of auxetic DAHs can achieve desirable vibroacoustic performance with a higher bending stiffness than conventional hexagonal honeycomb sandwich structures, and the design of gradient DAHs can be extended to obtain optimized vibration and noise-control capabilities.
\end{abstract}

\section{Introduction}

Metamaterials are artificial structures engineered to achieve unusual properties, and metamaterials that behave mechanically and have a negative Poisson's ratio (NPR) are called "auxetic" mechanical metamaterials [1-4]. These metamaterials expand rather than contract when stretched under uniaxial loading, differing from most natural materials. There have been important developments in many fields of auxetic metamaterials, including auxetic cellular solids of foams and honeycombs [5-7], microporous polymers [8], 3D re-entrants [9], chiral structures [10], and rotating rigid structures [11]. Compared to traditional materials, these auxetic structures have superior mechanical properties such as enhanced shear resistance, indentation resistance, and fracture toughness in various automotive, aerospace, biomedicine, and intelligent system applications [1-4].

In recent decades, cellular solids have been progressively employed [6,7], and auxetic foams and honeycombs have 
been used in numerous studies to explore the multiple functionalities of the cellular solid structures. Among the practical engineering applications involving cellular materials, sandwich structures (usually a simplified 2D sandwich beam) with in-plane (truss-like or prismatic) honeycomb cores have been explored for vibration and acoustic attenuation purposes, with the sound transmission loss (STL) frequently used as an evaluation metric [12-23]. These materials can be artificially tailored to create high-stiffness structures with low volumes and weights in contrast to cellular foams [13]. As a basis for the design of sandwiched core arrangements, previous scholars have reported that sandwich structures with embedded in-plane cellular cores transmit less sound than those with out-of-plane cellular cores [14], and core anisotropy can lead to a higher STL [15]. El-Raheb and Wagner [16] previously investigated the structural-acoustic performance of sandwich panels with periodic square trusslike cores. Ruzzene [17] analyzed the vibration and sound radiation performance of sandwich panels with in-plane hexagonal and re-entrant honeycomb cores, and Griese et al. [18] studied the effects of prismatic conventional and auxetic hexagonal honeycomb cell geometries on the sound transmission properties of sandwich panels. These studies indicated that sandwich panels with auxetic re-entrant cellular cores exhibit better sound insulation behavior than the conventional honeycomb sandwich structures with positive Poisson's ratios. Spadoni and Ruzzene [19] designed an auxetic chiral truss-core sandwich beam and explored its potential benefit in terms of vibration isolation and sound transmission reduction. To obtain an optimized STL, Denli and Sun [20] performed structural-acoustic optimizations of prismatic random cellular cores for minimum noise radiation in different frequency regions but without considering their manufacturability. Franco et al. [21] optimized sandwich panels with innovative cellular core configurations to minimize the sound radiation responses to various sources of excitation. As an extension of the study mentioned in [18], Galgalikar and Thompson [22] optimized hexagonal honeycomb sandwich panels for maximum STL and discovered that the STL response strongly depends on the numbers of unit cells in the horizontal and vertical directions in addition to the cell internal angles. Li and Yang [23] optimized the STL responses of sandwich panels with hybrid hexagonal honeycomb cores and suggested that hybrid core configurations constitute a broader platform for optimal acoustic designs. Apart from these direct structural-acoustic optimizations to obtain an optimized STL, topology optimization based on the band gaps of periodic metamaterials has been applied for sound and vibration control [13] because the stop bands through which plane waves cannot propagate can be obtained via the corresponding dispersion curves [24, 25].

The aforementioned sandwiched cellular structures are usually characterized by repeating unit cells with a fixed geometry. However, most current metamaterials are generally classified into two types based on their structural arrangements: homogenous (with uniform periodic structures) and inhomogeneous (with nonuniform nonperiodic structures) [26]. Lim [27] theoretically pioneered a nonperiodic functionally gradient beam to attain Poisson curving in 2002. Then, sandwich structures with combined conventional and re-entrant hexagonal metamaterials were investigated based on various characteristics, such as the specified objective deformations [28]; the strength and failure properties under bending, compressive and impact loads [29-31]; the dynamic behavior [32,33]; and the sound transmission performance $[23,34]$. Moreover, functionally gradient auxetic metamaterials based on other topologies, such as double arrowhead honeycombs (DAHs), have been developed [35-38] in recent years. These nonuniform metamaterials have attracted considerable interest due to their high-order functionality and broad designability.

Accordingly, a novel sandwich panel with cellular cores of auxetic DAHs is proposed and investigated with respect to its vibration and acoustic characteristics. To the best of our knowledge, such structures have not been previously investigated. In addition, gradient double arrowhead cellular cores are designed and implemented in the direction of the panel thickness based on the features of the nonuniform metamaterials. Programmable structural-acoustic optimization processes are then implemented to design a modifiedgradient DAH for an optimized sandwich panel that radiates less sound at different frequency regions. To precisely predict the structural vibroacoustic performance, the spectral element method (SEM) $[17,19]$ was employed to accurately compute the natural frequencies and dynamic responses of cellular structures at arbitrary frequencies with a largely reduced number of elements and few degrees of freedom (DOFs) [39, 40].

In the remainder of the paper, Section 2 describes the unit cell geometry and the configurations of the sandwich structures based on DAHs. Section 3 presents SEM formulations to compute the dynamic eigenfrequencies and responses and gives the computational procedures for sound radiation and transmission loss. Next, the vibroacoustic performance of the considered structures is discussed in Section 4. Moreover, a programmable structural-acoustic optimization process for maximum STLs is presented in Section 5. Finally, Section 6 concludes this study.

\section{Geometries of the Sandwich Panels with Uniform and Gradient DAH Cores}

2.1. Cell Geometry of Auxetic DAH Arrays. As shown in Figure 1, four parameters define the cell geometry of a standard DAH, where $\theta_{1}$ and $\theta_{2}$ denote the respective internal angles between the two inclined cell ribs and the vertical axis, $l$ is the height of the base triangle in the $x$ direction, and $t$ is the cell wall thickness. $L_{x}$ and $L_{y}$ are the cell dimensions, and each unit cell is associated with a rectangle that measures $L_{x} \times L_{y}$, where $L_{x}=2 l$. Within a continuous honeycomb array, the cell dimensions can be related to the parameters by

$$
\tan \theta_{1}^{(i+1)}=\frac{\left(L_{x} / 2\right)}{\left(L_{x} / 2\right) / \tan \theta_{2}^{(i)}-L_{y}}=\frac{\tan \theta_{2}^{(i)}}{1-2 \alpha \tan \theta_{2}^{(i)}},
$$

where the superscript $(i)$ denotes the $i$ th layer and $\alpha=L_{y} / L_{x}$ is the cell aspect ratio. The cell aspect ratio $\alpha$ determines the 


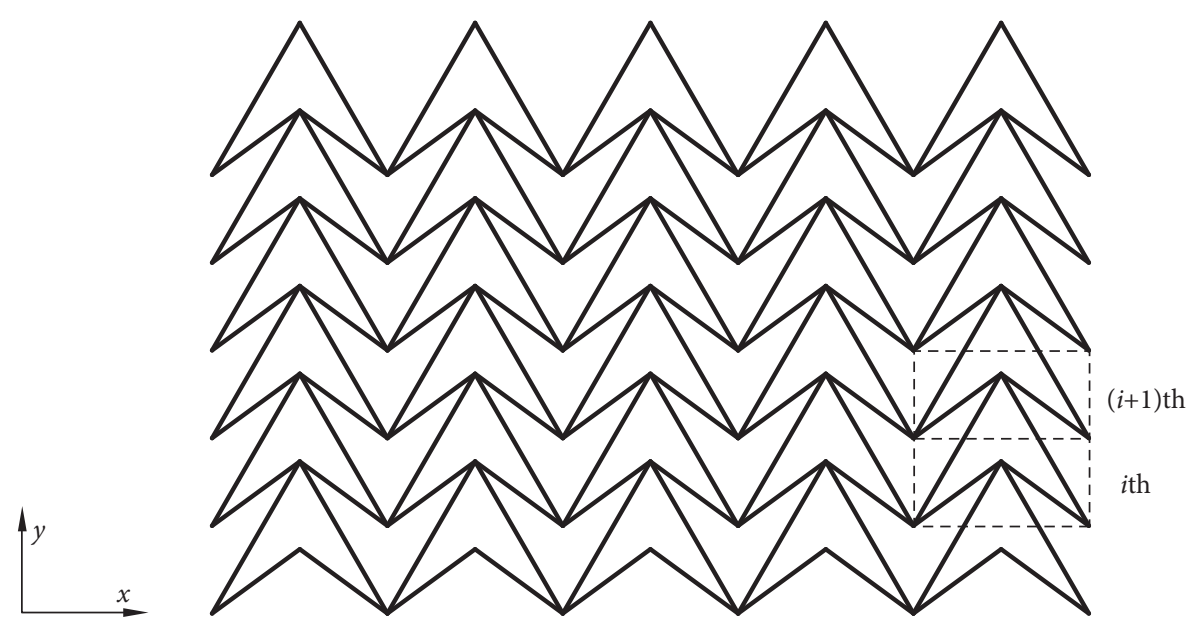

(a)

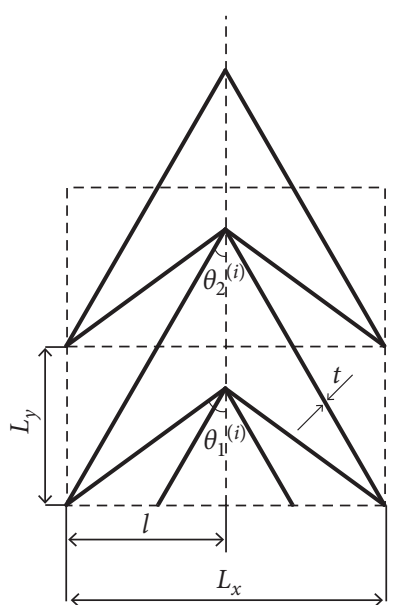

(b)

Figure 1: (a) Schematic diagrams of an array of auxetic DAHs and (b) a unit cell.

entire honeycomb core dimension, and the internal angle sequences $\boldsymbol{\Theta}_{1}$ and $\boldsymbol{\Theta}_{2}$ define the cell shape. For loading in the $y$ direction, the mechanical indices of Poisson's ratio and effective elastic modulus are given by Qiao and Chen [35]:

$$
\begin{aligned}
& v_{y x}^{*}=-\frac{\varepsilon_{x}}{\varepsilon_{y}}=-\frac{1}{\tan \theta_{1} \tan \theta_{2}}, \\
& \frac{E_{\mathrm{y}}^{*}}{E_{\mathrm{s}}}= \frac{1}{4}\left(\frac{t}{l}\right)^{3}\left[-\frac{4\left(\cos \theta_{1}-\cos \theta_{2}\right)}{\sin \theta_{1} \sin \theta_{2}}\right. \\
&-\frac{\left(\cos \theta_{1} \cos \theta_{2}-1\right)^{2}\left(\cos \theta_{1} \cos \theta_{2}-3\right)}{\sin \theta_{1} \sin \theta_{2}\left(\cos \theta_{1}-\cos \theta_{2}\right)} \\
&\left.+\frac{\left(\cos \theta_{1} \cos \theta_{2}+3\right)\left(\cos \theta_{1} \cos \theta_{2}-1\right)}{\cos \theta_{1}-\cos \theta_{2}}\right],
\end{aligned}
$$

where $E_{\mathrm{s}}$ is Young's modulus of the solid. To design a DAH cellular core, the angle sequence $\Theta_{2}$ is given, and $\Theta_{1}$ can then be derived through equation (1). The geometry of the honeycomb array is then determined and is scale-independent with a constant $L_{y} / L_{x}$. In addition, the feasible constraint of $\theta_{2}^{(i)}<$ $\arctan \left(l / L_{y}\right)$ must be imposed to attain the auxetic characteristics, yielding $\theta_{2}^{(i)}<\arctan (1 / 2 \alpha)$. The cell aspect ratio $\alpha$ utilized in this investigation is 0.2 , so that $\theta_{2}^{(i)}<68.2^{\circ}$. Here, the thicknesses of the cell wall and face sheets must be further considered to avoid excessive element distortion and overlap.

\subsection{Geometric Description of Sandwich Panels with Uniform} and Gradient DAH Cores. The considered sandwich panels with DAH cores are depicted in Figure 2. A DAH core is sandwiched between two constraining face sheets. Each model has an identical total length of $2 \mathrm{~m}$, and each cellular core comprises 40 cells in the length direction and 5 cells across the thickness of the core. $L_{x}$ is calculated to be $50 \mathrm{~mm}$ and $L_{y}$ is $10 \mathrm{~mm}$ with $\alpha=0.2$. However, the total thickness of each model differs because the unit cell in the top layer has a varying cell angle $\theta_{2}^{(i)}$. The constraint of $\theta_{2}^{(i)}>\arctan (1 / 5 \alpha)$ is then set to limit the influence resulting from the distinction of the panel thicknesses. Therefore, $\theta_{2}^{(i)}$ varies from 45 to $65^{\circ}$ without element overlap, and the total thickness ranges from 51.7 to $65 \mathrm{~mm}$.

To maintain a constant weight, the thickness of the two face sheets is constrained by a constant value of $t_{\mathrm{s}}=2 \mathrm{~mm}$. In addition, the thickness of the cellular cores is varied to maintain the weight equal to that of a baseline cellular sandwich panel with dimensions of $2 \mathrm{~m} \times 50 \mathrm{~mm}$ and a relative density of 0.1 . To this point in the study, both the overall length and weight have been held constant to compare the various properties of the sandwich models. The base material for the sandwich panel is aluminum with a Young's modulus $E_{\mathrm{s}}=71.9 \mathrm{GPa}$, Poisson's ratio $\nu=0.33$, and mass density $\rho_{\mathrm{s}}=2700 \mathrm{~kg} / \mathrm{m}^{3}$. The total weights of the two face sheets and core are $21.6 \mathrm{~kg} / \mathrm{m}$ and $27.0 \mathrm{~kg} / \mathrm{m}$, respectively.

The first three models shown in Figure 2(a) are defined as uniform DAH sandwich panels with identical unit cells. The mechanical indices $\left(\left|v_{y x}^{*}\right|\right.$ and $\left.E_{y}^{*}\right)$ are plotted in Figure 3(a) based on equations (2) and (3) as $\theta_{2}$ increases from 45 to $65^{\circ}$. Both of these values monotonously decrease as $\theta_{2}$ increases. Here, we stipulate that a large NPR signifies a Poisson's ratio with a large absolute value. Thus, the DAHs with larger NPRs yield large effective elastic modulus values. In contrast, as illustrated in Figures 2(b) and 2(c), the last two structures exhibit a varying pattern of DAHs across the panel thickness and are the so-called gradient DAH sandwich panels. As plotted in Figures 3(b) and 3(c), functionally increasing and decreasing sequences of $\Theta_{2}$ can generate sandwich models with DAHs of a decreasing NPR (DNPR) and an increasing NPR (INPR), respectively, particularly in this scenario. Regarding the gradient sandwich panels, the DAHs with large NPRs mainly yield large $E_{\mathrm{y}}^{*}$ values, as do the uniform sandwich panels.

\section{Theories of Vibration and Acoustic Analysis for DAH Cores}

3.1. SEM for the Vibration of Cellular Structures. The considered sandwich panel is assumed to be infinite along the $z$-axis as a simplified 2D planar strain problem, following the method of modeling prismatic cellular cores in references 


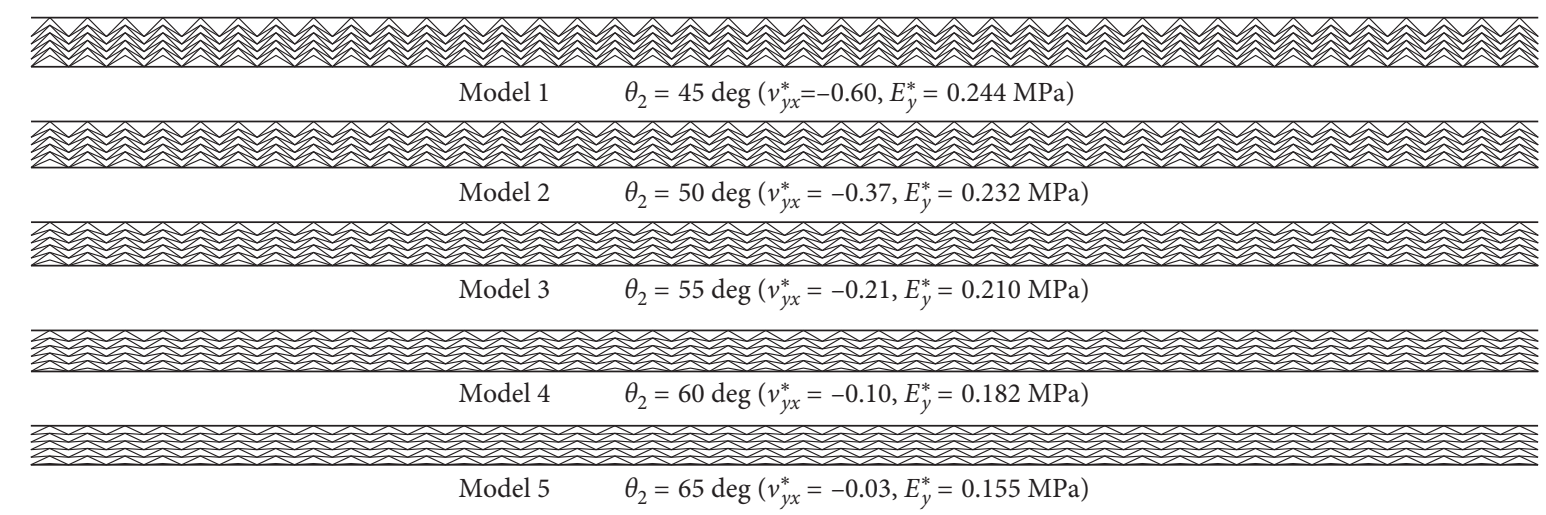

(a)

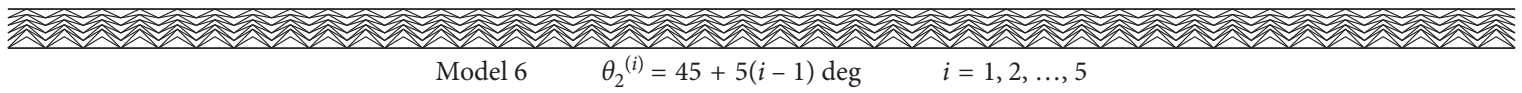

(b)
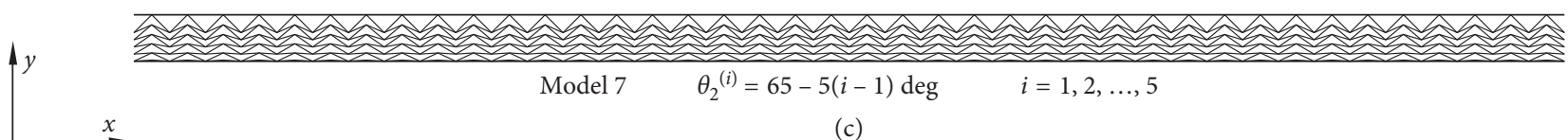

(c)

FIGURE 2: Sandwich panels with DAH cores from models 1 to 7: (a) the uniform models; (b) the gradient DNPR models; (c) the gradient INPR models.

[17-20, 22, 23, 40]. Therefore, the in-plane cellular structures are composed of rigidly connected beam elements treated as simplified 2D frames, and the long and thin frames are modeled as Bernoulli-Euler beams with an off-plane unit depth $[17,23,40]$. As shown in Figure 4, these elements are oriented in the $x O y$ plane, and the element displacement is described in the local coordinate system $(\xi, \psi)$ that rotates with respect to the global coordinate system $(x, y)$ at an angle $\varphi^{\prime}$. The DOFs of each element are $u$ and $w$, which represent the longitudinal and transverse displacements, respectively.

The partial differential equations of motion that describe the longitudinal and transverse vibrations of a uniform Bernoulli-Euler beam are represented in $[17,18,23,39,40]$ :

$$
\begin{aligned}
E A u_{\xi \xi}-\rho A u_{\mathrm{tt}} & =0, \\
E I w_{\xi \xi \xi \xi}+\rho A w_{\mathrm{tt}} & =0,
\end{aligned}
$$

where $E$ and $\rho$ are Young's modulus and mass density of the solid materials and $A$ and $I$ are the sectional area and moment of inertia of the beam, respectively. Note that $E=E_{\mathrm{s}} /\left(1-v^{2}\right)$ here under the planar strain assumption. Equation (4) can be simplified to an ordinary differential equation of harmonic motion at a circular frequency $\omega$ as

$$
\begin{aligned}
E A U_{\xi \xi}+\omega^{2} \rho A U & =0, \\
E I W_{\xi \xi \xi \xi}-\omega^{2} \rho A W & =0 .
\end{aligned}
$$

The homogeneous solution to equation (5) can be obtained in the following form:

$$
\begin{aligned}
U(\xi ; \omega) & =a_{1} e^{-i k_{\mathrm{L}} \xi}+a_{2} e^{+i k_{\mathrm{L}} \xi}, \\
W(\xi ; \omega) & =a_{3} e^{-i k_{\mathrm{F}} \xi}+a_{4} e^{-k_{\mathrm{F}} \xi}+a_{5} e^{+i k_{\mathrm{F}} \xi}+a_{6} e^{+k_{\mathrm{F}} \xi} .
\end{aligned}
$$

In this case, $a_{i}(i=1, \ldots, 6)$ are integration constants and

$$
\begin{aligned}
& k_{\mathrm{L}}=\sqrt{\frac{\rho A \omega^{2}}{E A}}, \\
& k_{\mathrm{F}}=\sqrt[4]{\frac{\rho A \omega^{2}}{E I}},
\end{aligned}
$$

where $k_{\mathrm{L}}$ and $k_{\mathrm{F}}$ are the wavenumbers of the longitudinal and transverse elastic waves, respectively. The spectral nodal displacement vector d comprises the longitudinal displacements, transverse displacements, and slopes of the frame elements and can be represented by

$$
\mathbf{d}=\left\{U_{i} W_{i} U_{\xi i} U_{j} W_{j} U_{\xi j}\right\}^{T}=\left\{U_{i} W_{i} \Theta_{i} U_{j} W_{j} \Theta_{j}\right\}^{T},
$$

where $i$ and $j$ denote the initial and final nodes of the beam element, as shown in Figure 4. Based on the displacements at the element boundaries ( $\xi=0$ and $\left.\xi=L^{(\mathrm{e})}\right)$, equation (6) expresses the integration constant $a_{i}$ with respect to d, which finally yields

$$
\mathbf{U}(\xi ; \omega)=\{U(\xi ; \omega) W(\xi ; \omega)\}^{T}=\mathbf{N}(\xi ; \omega) \mathbf{d},
$$

where $\mathbf{U}(\xi ; \omega)$ is the continuous displacement field and $\mathbf{N}(\xi$; $\omega)$ is the matrix of the transcendental dynamic shape functions. Following the sign convention shown in Figure 5, the spectral components of the longitudinal tensile forces $N$, bending moments $M$, and transverse shear forces $Q$ can be connected with $U(x)$ and $W(x)$ by

$$
\begin{aligned}
N(\xi) & =E A U^{\prime}(\xi), \\
M(\xi) & =E I W^{\prime \prime}(\xi), \\
Q(\xi) & =-E I W^{\prime \prime \prime}(\xi) .
\end{aligned}
$$




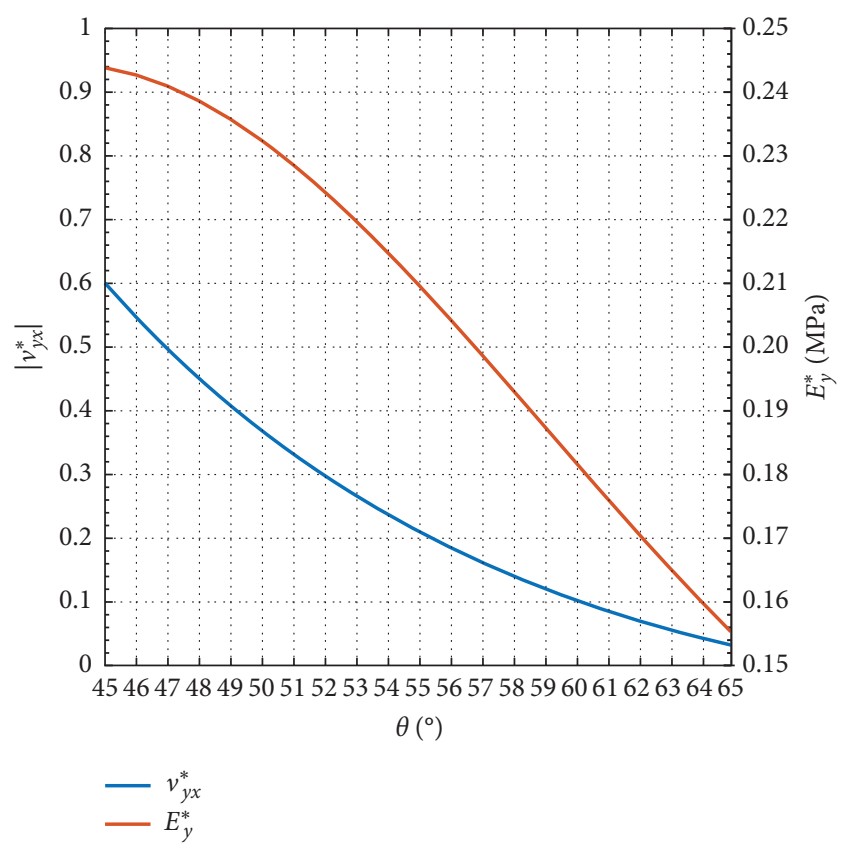

(a)

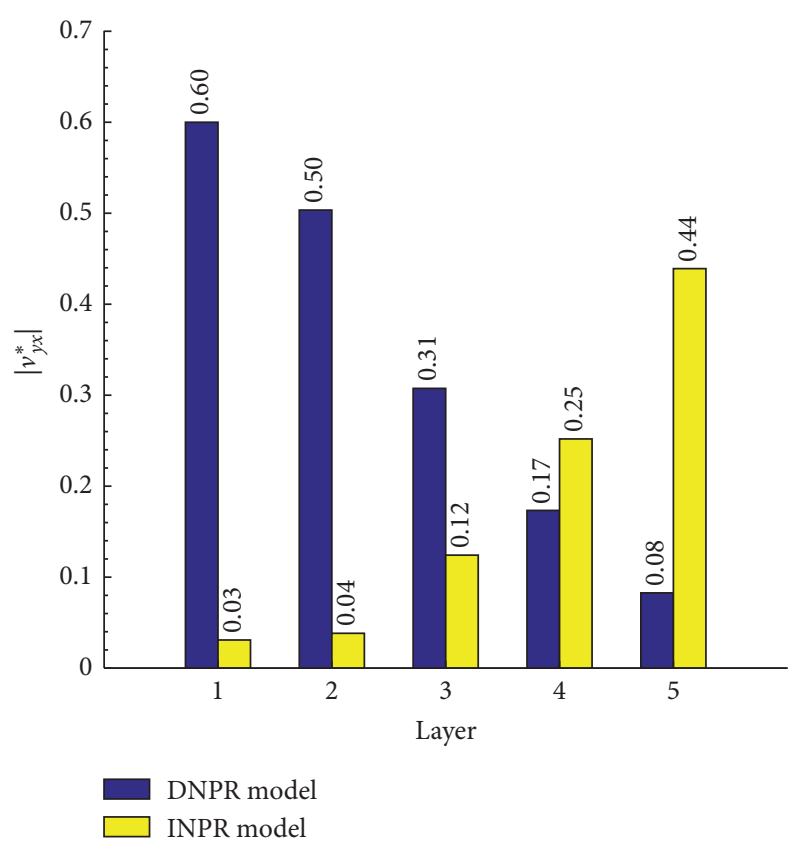

(b)

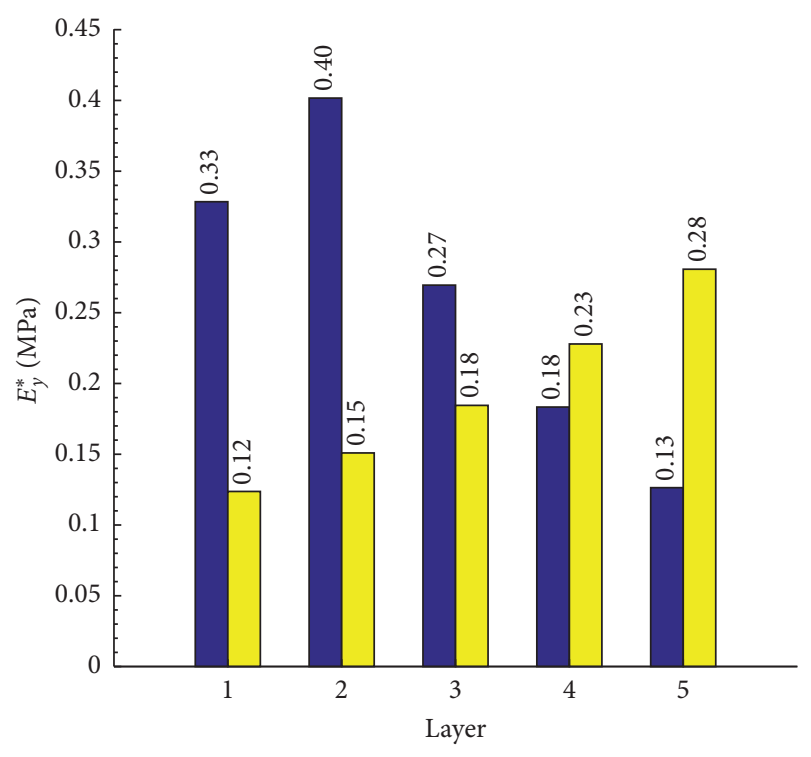

DNPR model

INPR model

(c)

Figure 3: Mechanical indices: (a) $\left|v_{y x}^{*}\right|$ and $E_{\mathrm{y}}^{*}$ within the uniform DAH sandwich models; (b) $\left|v_{y x}^{*}\right|$ within gradient DAH sandwich models 6 and 7 ; (c) $E_{\mathrm{y}}^{*}$ within gradient DAH sandwich models 6 and 7.

The spectral nodal longitudinal tensile force, transverse shear force, and bending moment can be related to the corresponding force and moment matrix $\mathbf{f}$ by

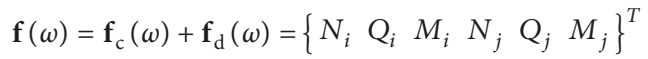

$$
\begin{aligned}
& =\left\{-N(0)-Q(0)-M(0)+N\left(L^{(\mathrm{e})}\right)+Q\left(L^{(\mathrm{e})}\right)+M\left(L^{(\mathrm{e})}\right)\right\}^{T} \text {, }
\end{aligned}
$$

where the nodal force vector $\mathbf{f}_{\mathrm{c}}(\omega)$ is associated with the concentrated dynamic forces that are directly applied at the spectral nodes, and $\mathbf{f}_{\mathrm{d}}(\omega)$ is associated with the distributed dynamic forces. A spectral distributed load $\mathbf{P}(x ; \omega)$ must be transferred to each node of the element by

$$
\mathbf{f}_{\mathrm{d}}=\int_{0}^{L} \mathbf{N}^{T}(\xi ; \omega) \mathbf{P}(\xi ; \omega) d \xi
$$

Substituting equation (9) into (10) and implementing the results into the right-hand side of equation (11), we obtain 


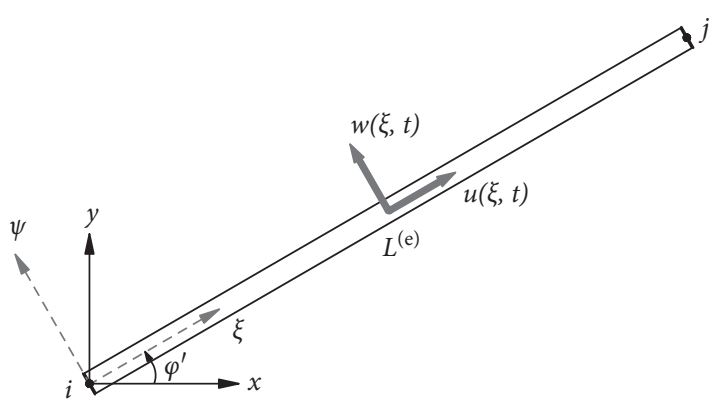

FIGURE 4: Global and local coordinate systems and DOFs, where $(L)^{(\mathrm{e})}$ denotes the element length.

$$
\mathbf{S}(\omega) \mathbf{d}=\mathbf{f}(\omega)
$$

where $\mathbf{S}(\omega)$ is the symmetric matrix of general spectral framed elements. Equation (13) must be transformed from the local coordinate system to the global coordinate system using the coordinate transformation matrix $\mathbf{T}$ :

$$
\mathbf{S}_{\mathrm{g}}(\omega) \mathbf{d}_{\mathrm{g}}=\mathbf{f}_{\mathrm{g}}(\omega)
$$

where

$$
\mathbf{S}_{\mathrm{g}}(\omega)=\mathbf{T}^{-1} \mathbf{S}(\omega) \mathbf{T}
$$

Finally, the spectral equation for each element is assembled, and the spectral equation of the entire system is given by

$$
\overline{\mathbf{S}}_{\mathrm{g}}(\omega) \overline{\mathbf{d}}_{\mathrm{g}}=\overline{\mathbf{f}}_{\mathrm{g}}(\omega)
$$

where $\overline{\mathbf{S}}_{g}$ is the overall spectral element matrix, $\overline{\mathbf{d}}_{\mathrm{g}}$ is the overall nodal displacement vector, and $\overline{\mathbf{f}}_{\mathrm{g}}$ is the overall nodal force vector.

Then, the eigenvalue problem for a spectral element structural system can be reduced from equation (16) to

$$
\overline{\mathbf{S}}_{\mathrm{g}}(\omega) \overline{\mathbf{d}}_{\mathrm{g}}=0,
$$

and the infinite eigenfrequencies $\omega_{i}$ can be determined using the condition that

$$
\operatorname{det} \overline{\mathbf{S}}_{\mathrm{g}}\left(\omega_{i}\right)=0,
$$

at $\omega=\omega_{i}$. Here, $\overline{\mathbf{S}}_{\mathrm{g}}\left(\omega^{*}\right)$ is a transcendental function of frequency $\omega$, and thus equation (18) is a transcendental eigenvalue problem with exact eigensolutions. The Wittrick-Williams (W-W) algorithm [41-43] is employed here to search for the eigenfrequencies without missing any eigenvalues. In this algorithm, the total number of eigenfrequencies below an arbitrarily chosen frequency $\omega^{*}$ can be given by

$$
J\left(\omega^{*}\right)=J_{\mathrm{c}}\left(\omega^{*}\right)+s\left[\overline{\mathbf{S}}_{\mathrm{g}}\left(\omega^{*}\right)\right],
$$

where $J\left(\omega^{*}\right)$ is the total number of eigenfrequencies below $\omega^{*}, s\left[\overline{\mathbf{S}}_{\mathrm{g}}\left(\omega^{*}\right)\right]$ is the sign count of $\overline{\mathbf{S}}_{\mathrm{g}}\left(\omega^{*}\right)$ and is computed as the number of negative elements on the diagonal of the upper triangular matrix of $\overline{\mathbf{S}}_{\mathrm{g}}\left(\omega^{*}\right)$ that is obtained by Doolittle decomposition. $J_{c}\left(\omega^{*}\right)$ is the total number of eigenfrequencies below $\omega^{*}$ under the condition that all displacements $\overline{\mathbf{d}}_{\mathrm{g}}$ are constrained to zero. $J_{\mathrm{c}}\left(\omega^{*}\right)$ for simplified planar frame elements can be obtained from

$$
J_{\mathrm{c}}\left(\omega^{*}\right)=\sum_{j=1}^{N}\left[J_{\mathrm{L} j}\left(\omega^{*}\right)+J_{\mathrm{F} j}\left(\omega^{*}\right)\right],
$$

after dividing the complete structure into $N$ frame members with fixed ends, where $J_{\mathrm{L} j}\left(\omega^{*}\right)$ and $J_{\mathrm{F} j}\left(\omega^{*}\right)$ are the numbers of the longitudinal and flexural eigenfrequencies below $\omega^{*}$ for the $j$ th frame member with fixed boundaries, respectively. The iteration procedure implemented to compute the $r$ th eigenfrequency below $\omega^{*}$ resembles the dichotomies algorithm, which was detailed in the study of Li and Yang [23].

For a significantly minimized system scale, the total numbers of nodes and elements are 447 and 881 for each sandwich model, respectively. Since the SEM can provide exact frequency-domain solutions without refined mesh discretization as the frequency increases [39], the mesh connections remain unchanged regardless of the material properties, structure size, or targeted frequencies. Although the spectral matrices in equation (16) need repeated assembly and computation at each frequency step, the SEM still provides an extremely accurate and time-saving alternative for dynamic problems involving cellular sandwich structures.

3.2. Sound Radiation and Transmission Loss Analysis. The considered sandwich model with DAH cores is exposed to a planar incident wave of acoustic pressure on the bottom face sheet as shown in Figure 6. The model is located between two rigid baffles of infinite length. The vibration of the bottom face sheet is transmitted through the cellular core to the upper face sheet; then, the sandwich structure radiates sound in the fluid domain. The ends of the structure, both ends of the face sheets, and the cellular core ribs adjacent to the ends, are all constrained in the $x$ and $y$ directions with pinned boundary conditions. The contribution of the airborne transmission to the total radiated sound power is neglected because of the high structural stiffness $[40,44]$, and thus only the structure-borne transmission is considered by following most of the relevant studies referred in Section 1.

The acoustic incident wave is harmonic with a unit amplitude $\left|p_{\mathrm{i}}\right|=1 \mathrm{~Pa}$ and an incidence angle $\alpha$ with respect to the $y$-axis. The incident sound power $W_{\mathrm{i}}$ over the bottom face sheet is given by

$$
W_{\mathrm{i}}=\frac{\left|p_{\mathrm{i}}\right|^{2} L_{\mathrm{B}} \cos \alpha}{2 \rho_{\mathrm{a}} c_{\mathrm{a}}},
$$

where $\rho_{\mathrm{a}}$ and $c_{\mathrm{a}}$ represent the fluid density and sound speed, respectively, and $L_{\mathrm{B}}$ represents the bottom face sheet. The spectral nodal force can be obtained by equation (12). The transmitted sound pressure $p_{\mathrm{t}}\left(\mathbf{r}^{\prime}\right) e^{-j \omega t}$ at an observation point $\mathbf{r}^{\prime}$ can be computed by Rayleigh's first integral for onedimensional baffled radiators in two dimensions [45]:

$$
p_{\mathrm{t}}\left(\mathbf{r}^{\prime}\right)=\frac{\rho_{\mathrm{a}} c_{\mathrm{a}} k}{2} \sum_{L_{\mathrm{U}}} \int_{0}^{L_{i}^{(e)}} v(\mathbf{r}) H_{0}^{(1)}\left(k\left|\mathbf{r}-\mathbf{r}^{\prime}\right|\right) d \xi
$$

where $j$ is an imaginary unit, $k=\omega / c_{\mathrm{a}}$ is the acoustic wavenumber, $\mathbf{r}$ is the position of the fluid particles on the upper face sheet $L_{\mathrm{U}}$ with a normal velocity $v(\mathbf{r}),\left|\mathbf{r}-\mathbf{r}^{\prime}\right|$ is the distance between the fluid particle and observation point, and $H_{0}^{(1)}$ 


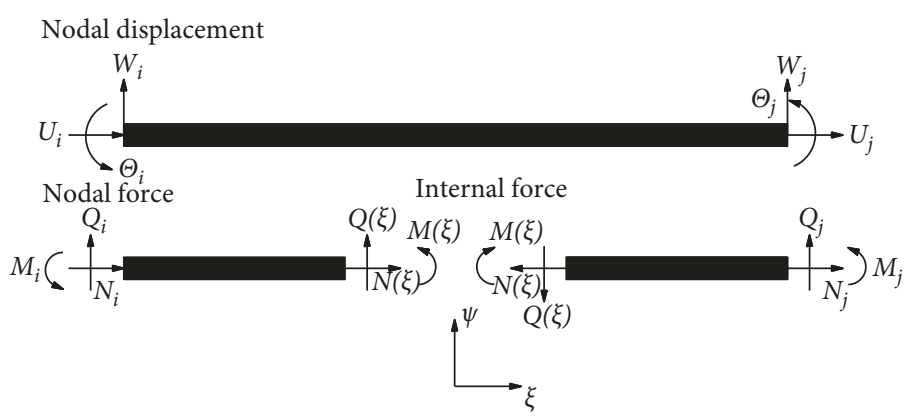

Figure 5: Sign convention for a Bernoulli-Euler beam element.

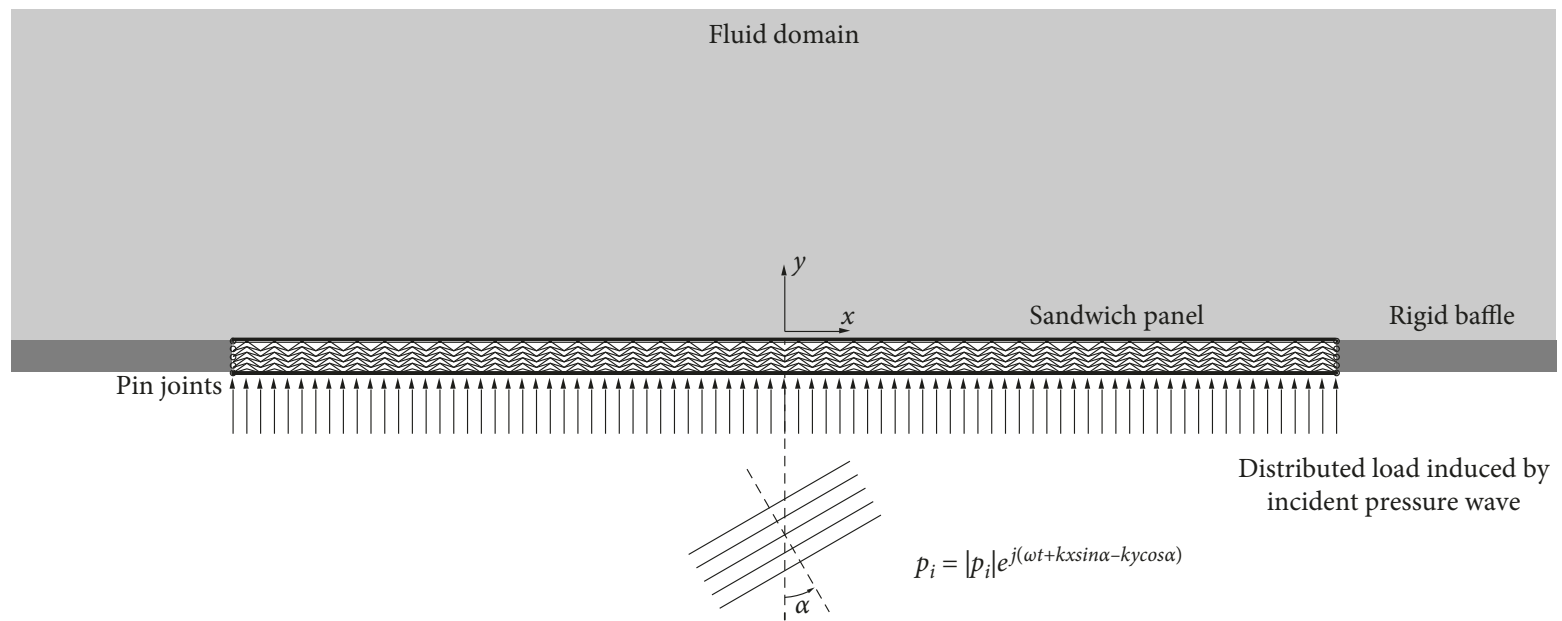

Figure 6: Acoustic loading and boundary conditions for a baffled, simply supported sandwich panel with a DAH core.

denotes a Hankel function of the first kind. The transmitted sound power $W_{\mathrm{t}}$ can be derived via integration over the upper face sheet $[17-19,21-23]$ by substituting equation (22) into

$$
W_{\mathrm{t}}=\frac{1}{2} \operatorname{Re} \int_{L_{\mathrm{U}}} p_{\mathrm{t}}\left(\mathbf{r}^{\prime}\right) v^{*}\left(\mathbf{r}^{\prime}\right) d \xi=\frac{1}{2 \rho_{\mathrm{a}} c_{\mathrm{a}}} \int_{L_{\mathrm{U}}}\left|p_{\mathrm{t}}^{2}\left(\mathbf{r}^{\prime}\right)\right| d \xi,
$$

where * denotes the complex conjugate. The spectral element shape functions are transcendental in the integrands, and a fifth-order Gaussian quadrature is used to compute the numerical integral of the radiated power with a compromise between computational accuracy and efficiency. The angle of wave incidence $\left(\alpha=0^{\circ}\right)$ is specified for simplicity in this study. Accordingly, the transmission loss is defined as

$$
\mathrm{STL}=10 \log _{10}\left(\frac{W_{\mathrm{i}}}{W_{\mathrm{t}}}\right) \text {. }
$$

The STL characteristics of a panel can be generally divided into four distinct regions: stiffness, resonance, mass, and coincidence regions from low to high frequencies $[18,25]$. Moreover, the theoretical STL of an infinitely long and thin panel was estimated by Griese et al. [18]:

$$
\begin{aligned}
\operatorname{STL}(\omega) & =10 \log _{10}\left[1+\left(\frac{m_{\mathrm{p}} \omega \cos \alpha}{2 \rho_{\mathrm{a}} c_{\mathrm{a}}}\right)^{2}\right] \\
& \approx 20 \log _{10}\left(m_{\mathrm{p}} f\right)-42,
\end{aligned}
$$

where $m_{\mathrm{p}}$ is the mass per unit surface area of the panel and $f$ is the frequency. The final expression is known as the mass law for sound transmission loss under a normal planar incident wave when $\alpha=0^{\circ}$ and $m_{\mathrm{p}}=48.6 \mathrm{~kg} / \mathrm{m}$ for the considered models that are assumed as single panels in this scenario.

\section{Vibration and Sound Transmission Performance of the Sandwich Panels with DAH Cores}

4.1. Natural Frequencies. The considered models are treated as undamped linear elastic systems because the effects of damping on natural frequency searching are generally small [39]. The sandwich panels are simply-supported by pin joints, as illustrated in Figure 6. The natural frequencies that were computed via the $\mathrm{W}-\mathrm{W}$ algorithm (SEM) are listed in Table 1 and plotted in Figure 7 for each considered model.

For the uniform models 1 to 5 , the fundamental frequency that represents the structural bending stiffness decreases as the feature angle $\theta_{2}$ of the DAHs increases. This result is associated with the low $E_{\mathrm{y}}^{*}$ of the unit cells with a large feature angle, as shown in Figure 3(a), and the deviations in the panel thicknesses also have an effect. However, the high-order natural frequencies of the uniform models with large $\theta_{2}$ values are larger than those of the 
TABLE 1: Series of the natural frequencies of the representative uniform and gradient DAH sandwich models via SEM (Hz).

\begin{tabular}{lcccccccccc}
\hline Model & 1st & 2nd & 3rd & 4th & 5th & 6th & 7th & 8th & 9th & 10th \\
\hline Model 1 & 88.8 & 210.5 & 357.8 & 516.1 & 677.5 & 784.2 & 836.4 & 910.0 & 923.1 & 928.0 \\
Model 2 & 86.6 & 210.6 & 364.3 & 532.4 & 706.7 & 856.6 & 881.2 & 1049.6 & 1129.2 & 1153.1 \\
Model 3 & 83.7 & 207.3 & 363.5 & 536.9 & 718.7 & 885.3 & 904.1 & 1081.8 & 1255.4 & 1333.3 \\
Model 4 & 80.6 & 201.9 & 357.0 & 530.6 & 713.6 & 882.0 & 902.6 & 1080.9 & 1257.4 & 1326.9 \\
Model 5 & 77.8 & 194.6 & 342.3 & 503.4 & 581.2 & 615.7 & 666.1 & 667.3 & 719.9 & 816.7 \\
Model 6 & 74.4 & 182.5 & 317.9 & 467.5 & 623.9 & 781.5 & 870.3 & 937.1 & 1087.5 & 1179.9 \\
Model 7 & 94.5 & 230.2 & 395.0 & 565.8 & 729.2 & 823.6 & 878.1 & 969.4 & 1003.0 & 1023.0 \\
\hline
\end{tabular}

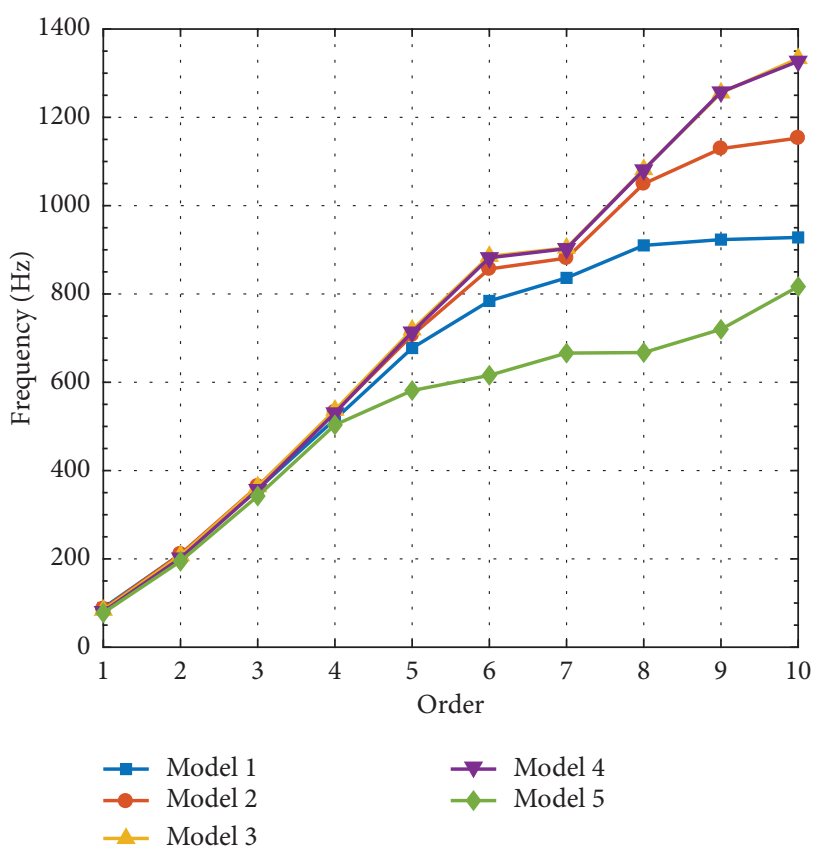

(a)

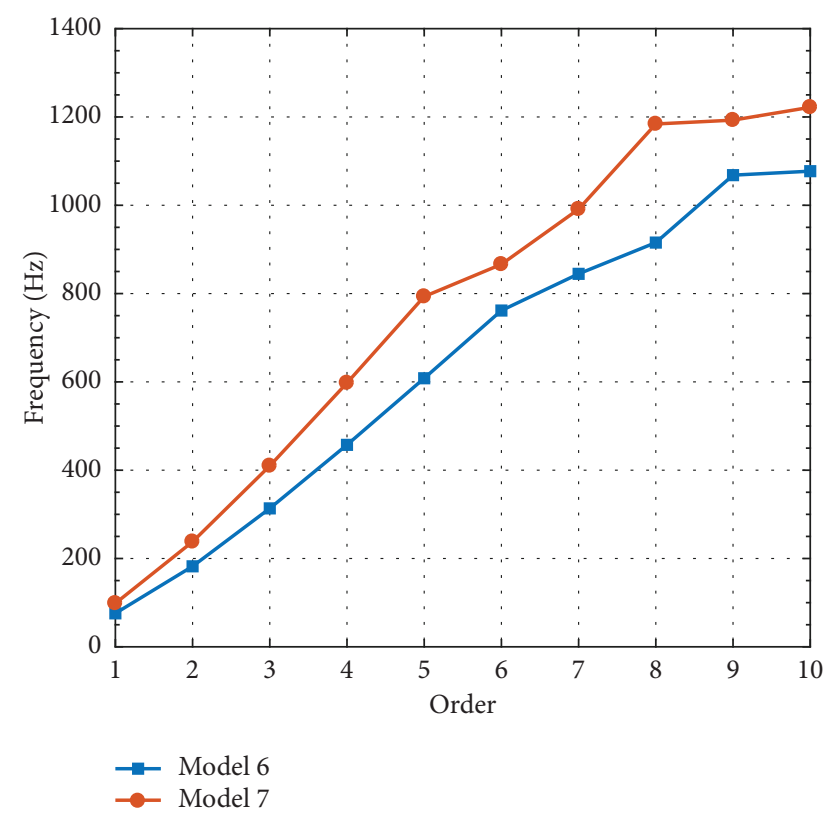

(b)

Figure 7: The first ten natural frequencies of the considered (a) uniform and (b) gradient DAH sandwich models.

models with small $\theta_{2}$ values until the feature angle exceeds $60^{\circ}$. This result implies that the static mechanical indices cannot completely reflect the structural dynamic behavior at high-order natural frequencies. Concerning the gradient models, the natural frequencies of model 7 are always higher than the frequencies of model 6 , mainly due to the large differences in the panel thickness.

The vibrating modes of the corresponding natural frequencies for the representative models are depicted in Figure 8. The modes of a global vibrating mode shape correspond to global natural frequencies, and the others correspond to local natural frequencies. The figures in the left column illustrate the third global bending modes when the entire sandwich panel bends in the form of a curved beam in two dimensions. The figures in the right column present the foremost local frequencies when the cellular cores are partly distorted. It can be observed that the foremost local frequencies occur when the initially straight curves become bended as plotted in Figure 7 . The varying core configurations of the considered models yield diverse mode shapes, and the gradient DAHs within nonuniform cellular cores further provide flexible vibrating deformation mechanisms.
4.2. Dynamic Responses and Sound Radiation. For dynamic responses, damping can be introduced by a complex modulus $E^{*}=E_{\mathrm{s}}(1+j \eta)$ with a structural damping factor $\eta=0.01$; in this case, aluminum damping is considered in addition to the damping effects of the hinged connections of the cell ribs [17]. The acoustic loading and boundary conditions are the same as illustrated in Figure 6, and the dynamic deformation of the considered models is depicted in Figure 9 at 500, 1000, and $1400 \mathrm{~Hz}$.

To visualize the sound radiation distributions after vibrating deformations as shown in Figure 9, the radiated sound pressure levels (SPLs) are also presented in Figure 10 for models 3 and 6 at 500,1000, and $1400 \mathrm{~Hz}$. The amplitude of the incident pressure is $1 \mathrm{~Pa}(94 \mathrm{~dB})$, and the reference pressure $p_{0}$ is $20 \mu \mathrm{Pa}$. As plotted in Figure 10, the fluid observation area ranges from nondimensionalized values of $x=-0.5$ to $x=0.5$ and from nondimensionalized values of $y=0$ to $y=1$. Additionally, the sandwich panels are located in the middle between $x=-0.1$ and $x=0.1$ at $y=0$.

4.3. Sound Transmission Loss. In this study, the STL responses of the considered models with varying tailored DAH cores 


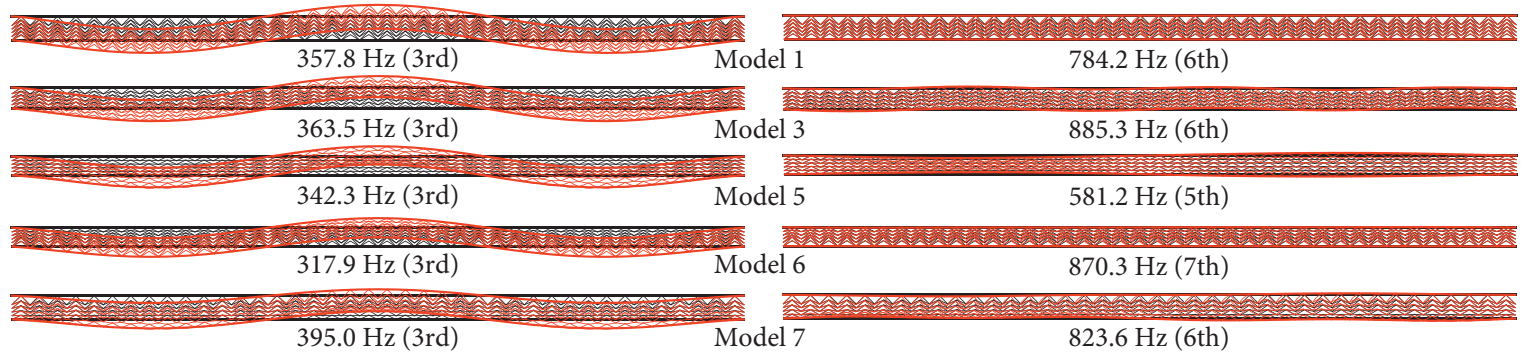

(a)

(b)

FIGURE 8: Vibrating mode shapes of the corresponding natural frequencies for the representative models: (a) the third bending global modes and (b) the foremost local modes.

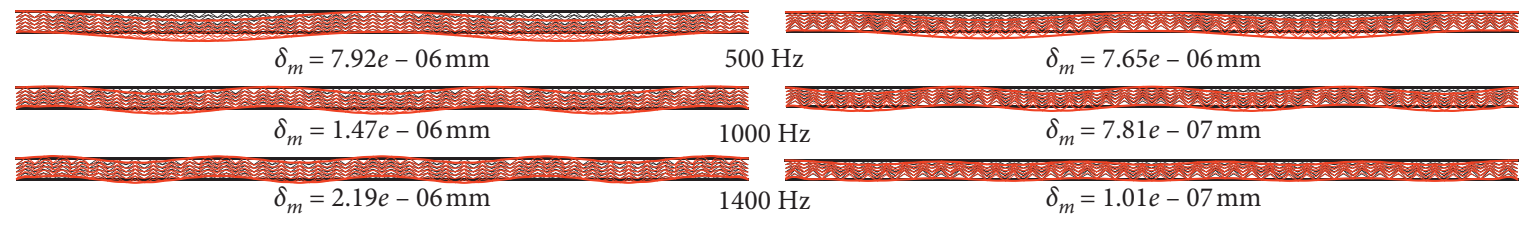

(a)

(b)

FIGURE 9: Vibrating deformations of the considered DAH sandwich models: (a) model 3 and (b) model 6 at 500, 1000, and 1400 Hz, where $\delta_{\mathrm{m}}$ denotes the displacement magnitude of the middle bottom face sheet.
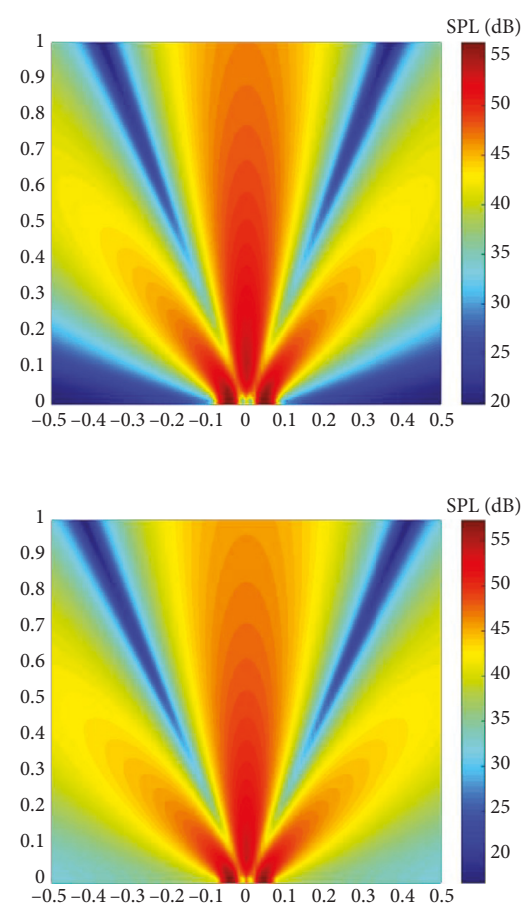

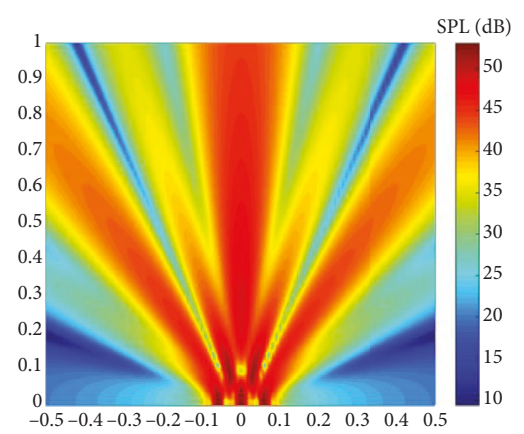

(a)

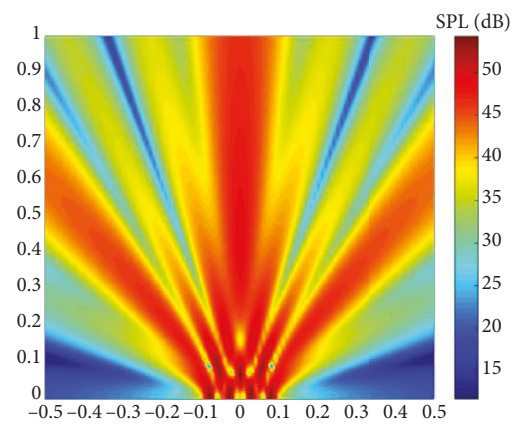

(b)
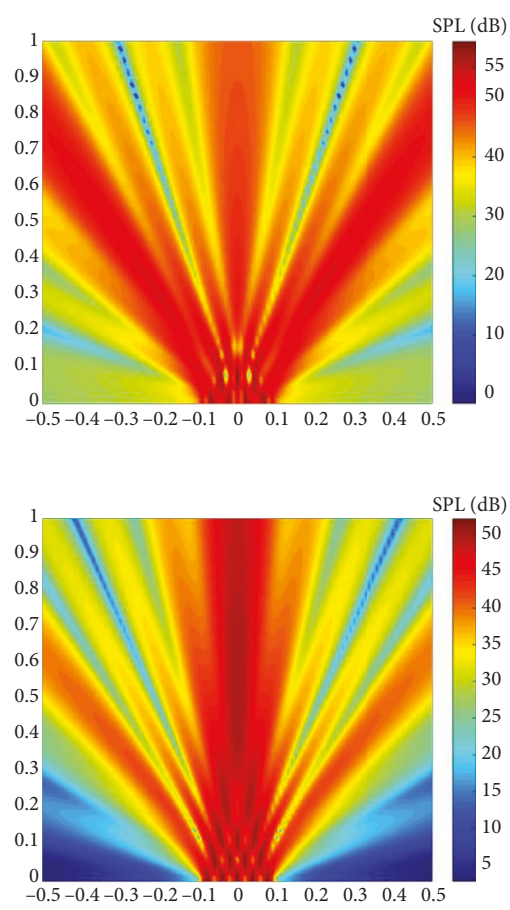

FIGURE 10: SPL distributions of the considered models: (a) model 3 and (b) model 5 at 500, 1000, and $1400 \mathrm{~Hz}$.

were primarily investigated in the region from 1 to $1500 \mathrm{~Hz}$, which covers the stiffness and resonance regions based on the natural frequencies discussed in Section 4.1. Thus, the acoustic performance at higher frequencies falls beyond the scope of this research. Two uniform hexagonal honeycomb sandwich models based on the configurations in $[18,22]$ are depicted in Figure 11, for comparison with the DAH sandwich panels. The compared hexagonal honeycomb sandwich models have the same unit cell arrangement of $40 \times 5$, overall dimensions of $2 \mathrm{~m} \times 50 \mathrm{~mm}$, and total weight of $m_{\mathrm{p}}=48.6 \mathrm{~kg} / \mathrm{m}$ for the baseline panel introduced in Section 2.2.

To verify the effectiveness and accuracy of the vibroacoustic calculation method in this paper, the numeric results compared with those of [18] are shown in Figure 12, which indicates that the sound transmission results via the proposed method agree quantitatively with those of the 


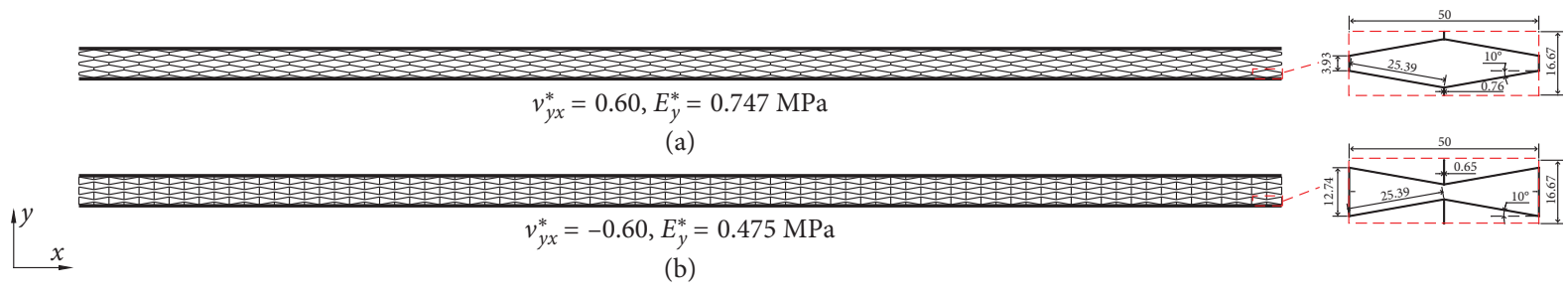

Figure 11: The compared sandwich models with uniform hexagonal honeycomb cores: cell feature angles of (a) $10^{\circ}$ and $(\mathrm{b})-10^{\circ}$.

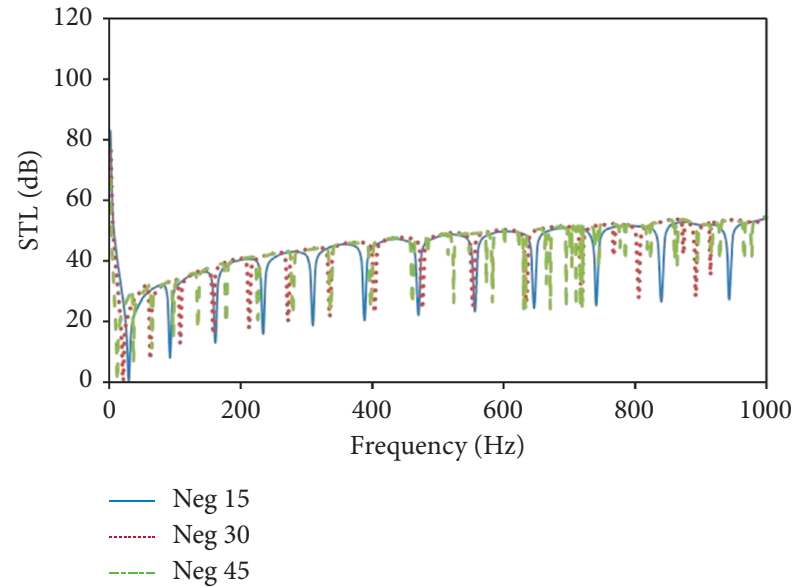

(a)

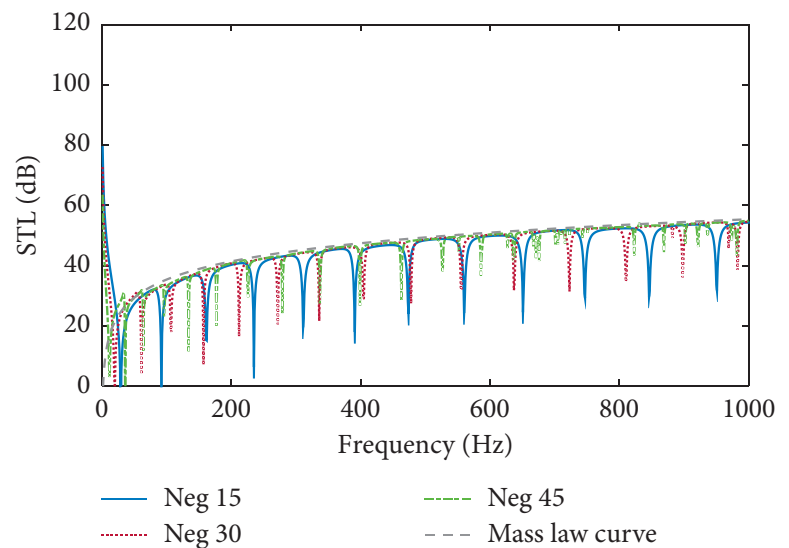

(b)

FIGURE 12: The calculated STLs of the sandwich panels for verification by comparing (a) Figure 11 in [18] and (b) the calculated STLs via the proposed method with the structural damping factor $\eta=0.001$.

published materials. Moreover, the STLs of the considered models from 1 to $1500 \mathrm{~Hz}$ are shown in Figures 13(a)-13(c) where the mass law trends are also plotted. The STL dips as the low frequencies align with the odd-order bending modes listed in Table 1 because the considered symmetric models strongly vibrate under symmetric excitation when odd bending resonance is encountered. The effective Young's modulus $E_{\mathrm{y}}^{*}$ is selected as a function of the panel stiffness following $[18,22]$. Although the DAHs in the considered models yield a smaller $E_{y}^{*}$ than the hexagonal honeycombs as shown in Figures 3 and 11, the modal density of the DAH sandwich models is much larger than the modal density of the sandwich panels with hexagonal cellular cores. This finding implies that sandwich panels with a DAH core yield a larger in-plane bending stiffness.

The overall average STL $\left(\mathrm{STL}_{\mathrm{o}}\right)$ of the considered models is shown in Figure 13(d) to assess the STL performance as an acoustic evaluation metric. A model that radiates less sound yields a larger $\mathrm{STL}_{\mathrm{o}}$. It can be concluded that a uniform sandwich model with a small $\theta_{2}$ or large NPR yields the best sound insulation performance, as shown in Figures 13(a) and 13(d). The STL $\mathrm{o}_{\mathrm{o}}$ of model 6 is larger than the STL of model 7, as shown in Figure 13(b), and the STL curve shapes of models 6 and 7 resemble the STL shapes of the uniform models (models 1 and 5) that feature the same $\theta_{2}$ for the bottom layer, respectively. As plotted in Figure 13(c), the $\mathrm{STL}_{\mathrm{o}}$ of model 9 with auxetic hexagonal honeycombs is larger than that of model 8, which is in agreement with the findings in $[17,18,22]$. Moreover, although both models 8 and 9 with conventional hexagonal honeycomb cores transmit less sound than the considered DAH sandwich models, their fundamental and high-order natural frequencies are far lower than those of the sandwich models with DAHs, as illustrated in Figure 13(c). This result indicates that the DAH sandwich panels have a stronger bending stiffness with desirable noise attenuation performance.

\section{Programmable Structural-Acoustic Optimization of the Gradient DAHs for Maximum STLs}

5.1. Mathematical Formulations for Structural-Acoustic Shape Optimizations. The structural-acoustic optimization problem is defined as

$$
\begin{array}{ll}
\text { find } & \boldsymbol{\Theta}_{2}, \\
\text { minimize } & -\mathrm{STL}_{\mathrm{a}}\left(\boldsymbol{\Theta}_{2} ; f_{1}, f_{2}\right) \\
\text { subject to } & \boldsymbol{\Theta}_{2}^{\mathrm{L}} \leq \boldsymbol{\Theta}_{2} \leq \boldsymbol{\Theta}_{2}^{\mathrm{U}} \\
& \pm\left(\boldsymbol{\Theta}_{2}^{(i+1)}-\boldsymbol{\Theta}_{2}^{(i)}\right) \leq 0, \\
& \pm\left(\boldsymbol{\Theta}_{2}^{(i)}-\boldsymbol{\Theta}_{2}^{(i+1)}\right) \leq \beta, \quad i=1,2, \ldots, N_{\mathrm{r}}-1, \\
& f_{0}\left(\boldsymbol{\Theta}_{2}\right)-f_{0} \leq 0,
\end{array}
$$

where $\boldsymbol{\Theta}_{2}^{\mathrm{L}}$ and $\boldsymbol{\Theta}_{2}^{\mathrm{U}}$ are the lower and upper bounding vectors of $\Theta_{2}$, respectively; $\operatorname{STL}_{\mathbf{a}}\left(\Theta_{2}\right)$ is the average STL between the 


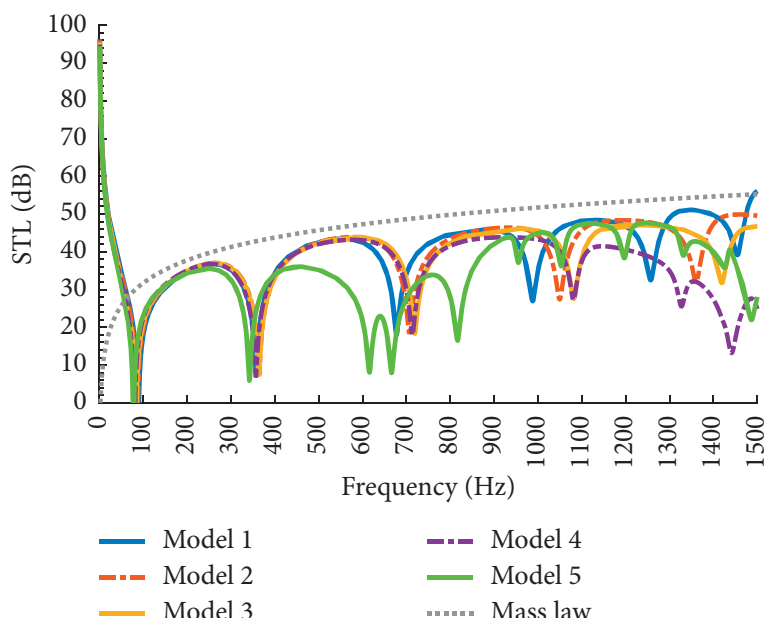

(a)

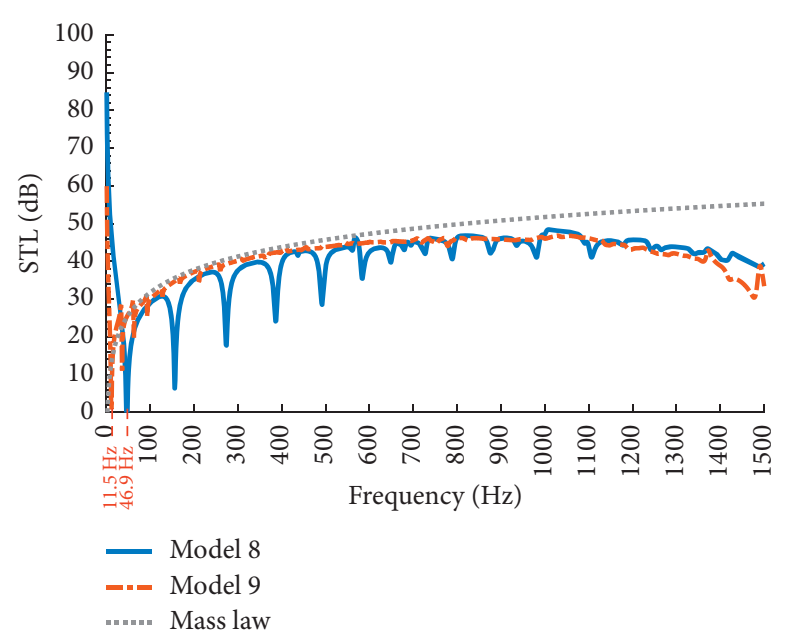

(c)

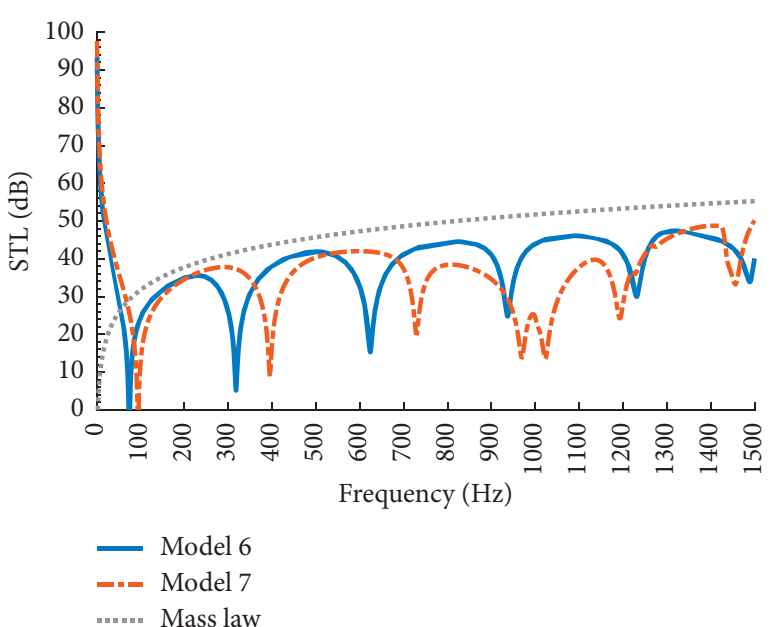

(b)

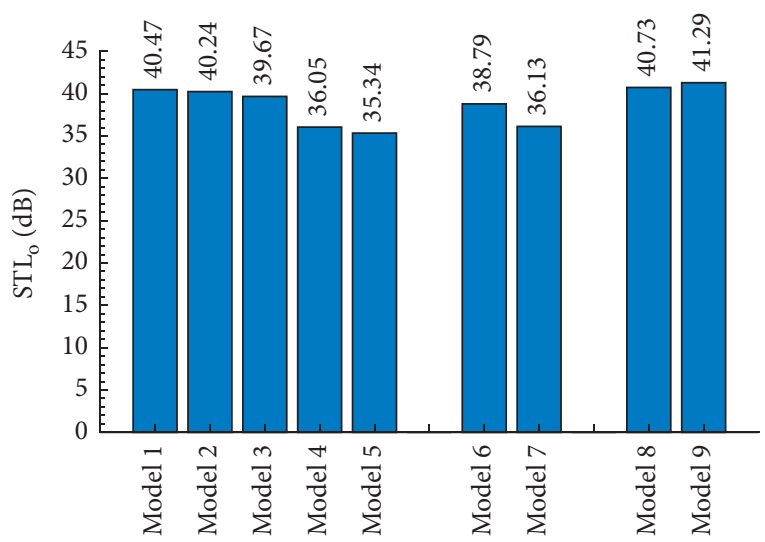

(d)

FIGURE 13: STLs of the representative sandwich models: (a) models 1 to 5; (b) models 6 and 7; (c) models 8 and 9. (d) The overall average STLs for the constant-weight sandwich models from 1 to $1500 \mathrm{~Hz}$.

specified frequencies of $f_{1}$ and $f_{2} ; f_{0}\left(\boldsymbol{\Theta}_{2}\right)$ is the fundamental frequency of the optimized model; $f_{0}$ is the minimum allowable natural frequency; and $\beta$ is the interval of $\theta_{2}^{(i)}$ set to avoid element overlap. In this scenario, $\Theta_{2}^{\mathrm{L}}=45^{\circ}, \Theta_{2}^{\mathrm{U}}=65^{\circ}$, and $\beta=10^{\circ}$. The second and third constraints limit the DAHs with gradient $\Theta_{2}$ sequences, where the positive sign represents a decreasing gradient $\boldsymbol{\Theta}_{2}$ (a gradient INPR) and the negative sign represents an increasing gradient $\boldsymbol{\Theta}_{2}$ (a gradient DNPR). The baseline model was employed with model 3 due to its moderate mechanical and acoustic performance, with values of $f_{0}=83.7 \mathrm{~Hz}$ and $\mathrm{STL}_{\mathrm{o}}=39.67 \mathrm{~dB}$.

The programmable design was achieved via a global optimization method based on the MultiStart algorithm. As a workflow [23], the MultiStart algorithm can automatically distribute the tasks in a problem and simultaneously generate a series of stochastic starting points for multiple processes or processors with parallel computing. Subsequently, the problems run independently from the corresponding start points, and the MultiStart algorithm finally combines the distinct local minima into a global vector to obtain a relatively optimized solution, where the chosen local solver involves sequential quadratic programming (SQP). In this scenario, the procedure was executed with $1 \times 10^{2}$ random start points, and the iteration tolerance for the local solver was $1 \times 10^{-3}$ to maintain computational efficiency and reasonability. The optimization procedures were executed for specified tonal and frequency band cases.

\subsection{Optimal Design for Specified Tonal and Frequency Band} Cases. Here, a tonal excitation at $1400 \mathrm{~Hz}$, which was close to an STL dip in the baseline model, was chosen for the tonal case. The STLs of the baseline and optimized gradient DAH sandwich models are compared in Figure 14(a), and the iterative curves converging to the optimum solutions are plotted in Figure 14(b). The configurations of the optimized models are shown in Figure 14(c), and the mechanical properties of the gradient DAH cores are given in Figure 14(d). In addition, the key parameters of the optimal design are listed and compared in Table 2, and both of the optimized models satisfy the high strength constraint and 


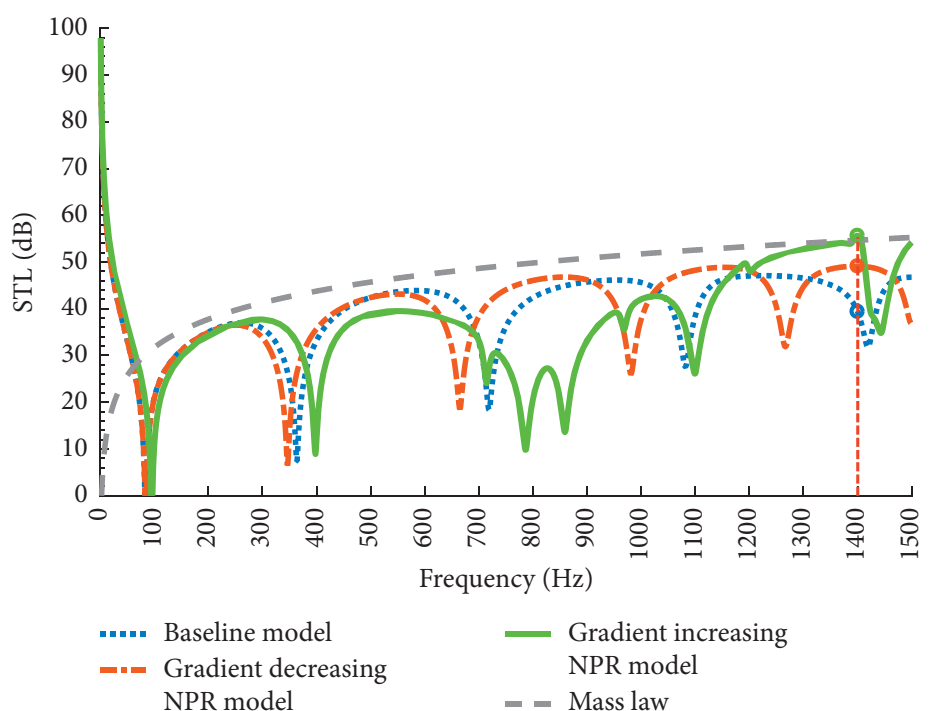

(a)

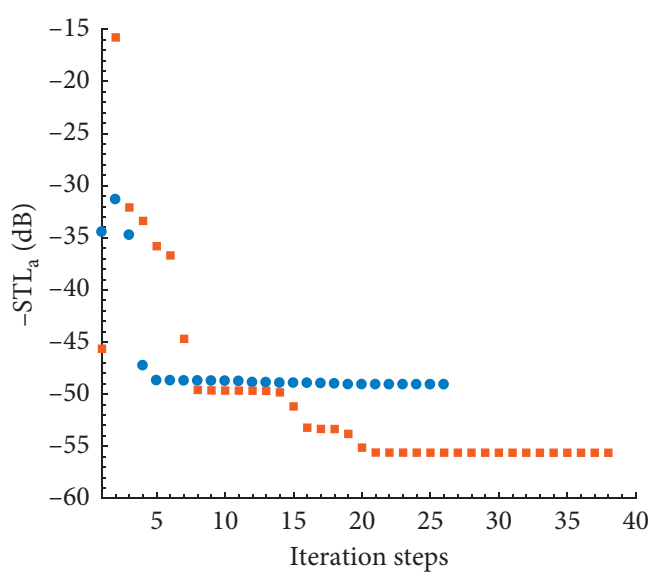

- Optimized gradient DNPR model

- Optimized gradient INPR model

(b)

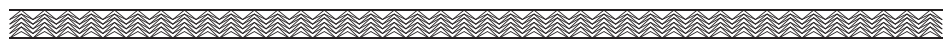

Optimized gradient DNPR model

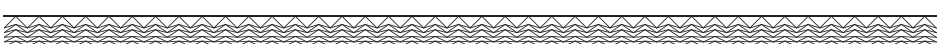

Optimized gradient INPR model

(c)

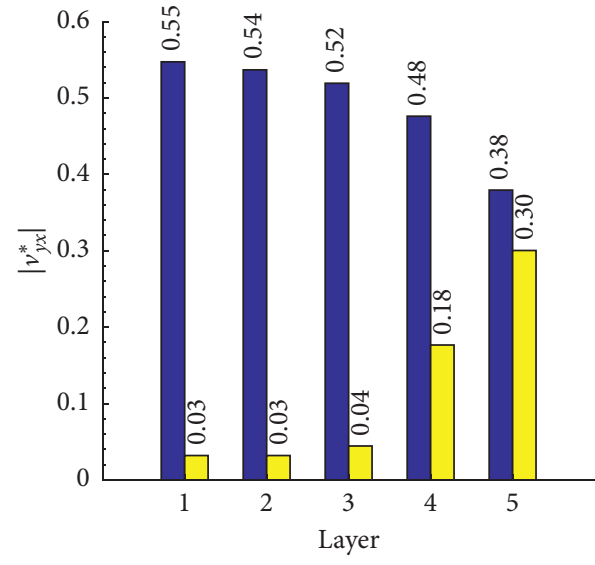

DNPR model

INPR model

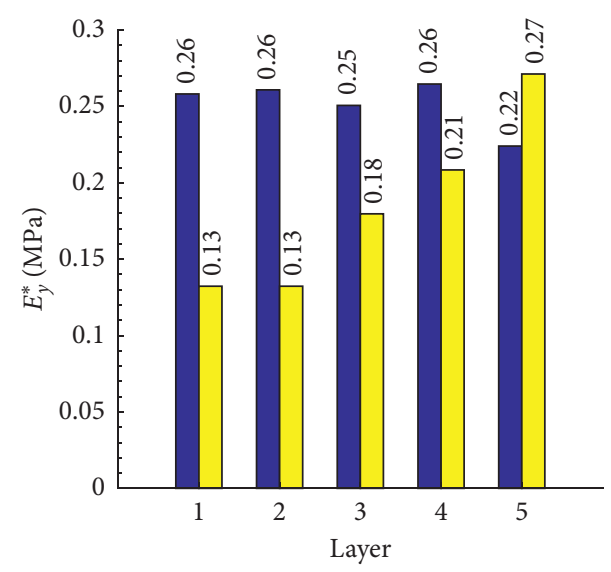

DNPR model

INPR model

(d)

FIGURE 14: Optimal design at $1400 \mathrm{~Hz}$ : (a) STLs of the baseline and optimized models; (b) iteration steps; (c) configurations of the optimized gradient NPR models; (d) mechanical properties of the DAHs in the optimized gradient NPR models.

TABLE 2: Characteristic parameters of the baseline and optimized models for optimal design at $1400 \mathrm{~Hz}$.

\begin{tabular}{lcccc}
\hline Model & $\boldsymbol{\Theta}_{2}(\mathrm{deg})$ & $\mathrm{STL}_{\mathrm{a}}(\mathrm{dB})$ & $f_{0}(\mathrm{~Hz})$ & $\mathrm{STL}_{\mathrm{o}}(\mathrm{dB})$ \\
\hline Baseline & {$[55.00,55.00,55.00,55.00,55.00]$} & 39.37 & 83.72 & 39.67 \\
Optimized gradient DNPR & {$[45.98,46.53,46.53,49.00,51.03]$} & 49.09 & 83.99 & 40.24 \\
Optimized gradient INPR & {$[64.91,64.91,56.90,54.96,45.09]$} & 55.61 & 95.27 & 37.68 \\
\hline
\end{tabular}

yield feasible core geometries. The $\mathrm{STL}_{\mathrm{a}}$ increments are approximately $9.72 \mathrm{~dB}$ for the optimized gradient DNPR model and $16.24 \mathrm{~dB}$ for the optimized gradient INPR model. The SPL distributions and dynamic deformations at $1400 \mathrm{~Hz}$ are illustrated in Figure 15 for the optimized models. As illustrated in Figures 15(b) and 14(a) for the optimized gradient INPR model, localized resonance occurs and the STL exceeds the mass law curve value at the target frequency. 


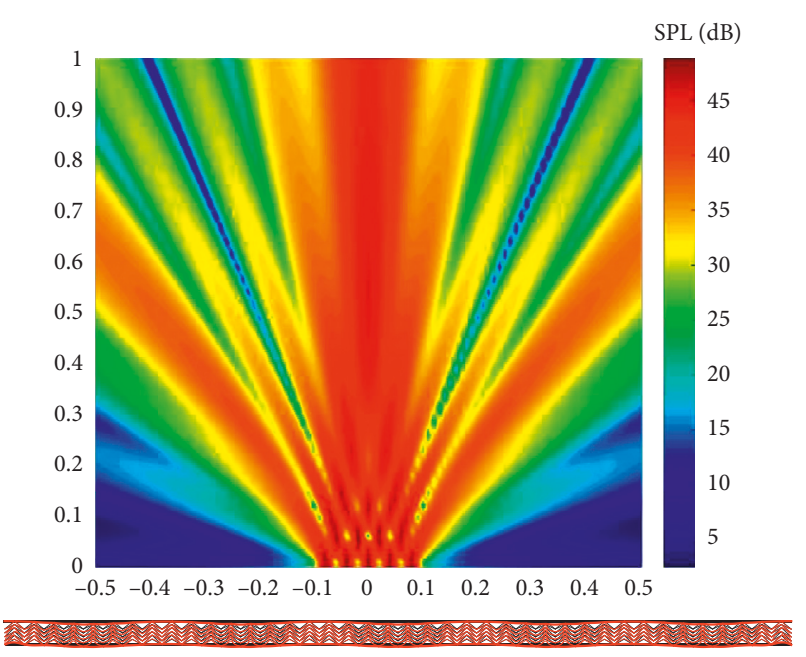

$\delta_{m}=1.82 e-07 \mathrm{~mm}$

(a)

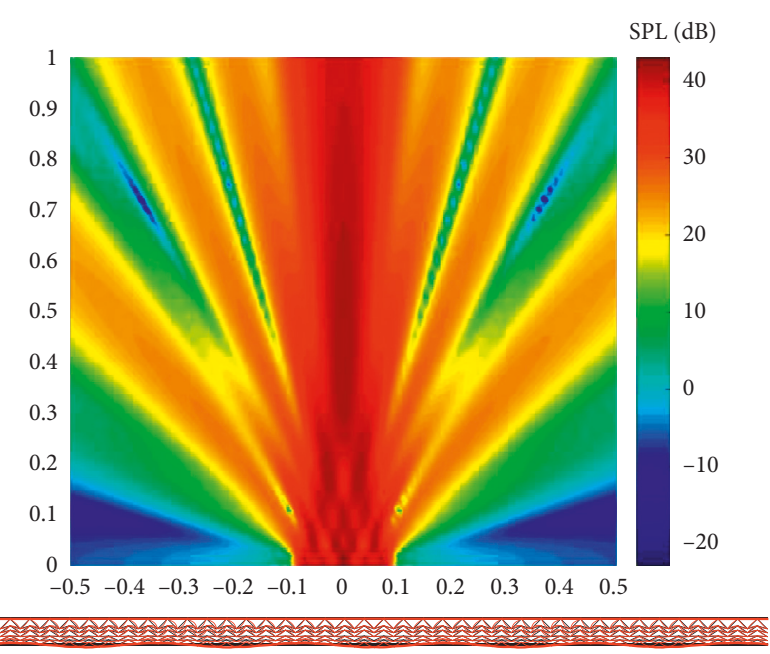

$\delta_{m}=1.96 e-06 \mathrm{~mm}$

(b)

FIGURE 15: SPL distributions and dynamic deformations of the optimized models at $1400 \mathrm{~Hz}$ (a) the optimized gradient DNPR model and (b) the optimized gradient INPR model.

The double arrowhead unit cells adjacent to the bottom face sheet absorb the majority of the vibrating energy; hence, the optimized gradient INPR model effectively transmits the least sound as a result.

Here, the chosen computational frequency interval was $20 \mathrm{~Hz}$ for the frequency band from 1000 to $1500 \mathrm{~Hz}$. The optimized information is depicted in Figure 16, and the characteristic parameters are listed in Table 3. The results demonstrate that the optimized solutions strictly satisfy the constraints of the bending stiffness and avoid element overlap. Furthermore, the reduction in average sound radiation power is approximately $2.51 \mathrm{~dB}$ for the optimized gradient DNPR model and $5.03 \mathrm{~dB}$ for the optimized gradient INPR model compared to the $\mathrm{STL}_{\mathrm{a}}$ of the baseline model. More optimized solutions could be obtained by increasing the number of start points, decreasing the iteration tolerance, or using larger integral orders.

\subsection{Comparison and Discussion of the Sound Transmission} Performance of the Optimized and Considered Models. The optimized gradient INPR models can achieve better insulation of radiated sound power than can the optimized gradient DNPR models, with improvements of $6.52 \mathrm{~dB}$ and $2.52 \mathrm{~dB}$ for the tonal and frequency band cases in this scenario, respectively. As plotted in Figures 14(d) and 16(d), the double arrowhead unit cells adjacent to the fluid domain feature a large $\left|\nu_{y x}^{*}\right|$ and yield a low $E_{y}^{*}$ in the gradient INPR models, and vice versa. The gradient INPR models enable a programmable DAH core for a high $\mathrm{STL}_{\mathrm{a}}$ that even exceeds the mass law curve values and yield a high bending stiffness; however, the use of gradient INPRs generally sacrifices overall sound insulation, with a lower $\mathrm{STL}_{\mathrm{o}}$. It can be concluded that the DAHs with large NPRs in upper layers improve the potential for obtaining an optimized STL within a specified frequency region, following the phenomenon that a uniform DAH sandwich model with a large NPR yields high sound insulation performance. Referring to the STL curves in Figures 14(a) and 16(a), the DAHs in the bottom layers mainly affect the STL curve shapes, as discussed in Section 4.3, and therefore, the overall noise insulation properties are limited.

\section{Conclusions}

Sandwich structures composed of uniform and gradient DAH cores across the panel thickness were presented, and the vibration and sound transmission properties of the proposed structures were evaluated by comparing uniform and gradient DNPR and INPR models, as well as uniform hexagonal honeycomb sandwich panels. The following conclusions were drawn from the study:

(1) Regarding the vibration properties, the various core configurations of the gradient sandwich DAH panels yield more diverse mode shapes and provide more flexible vibrating deformation mechanisms than do the uniform models.

(2) With respect to the sound transmission performance with normal incidence, the uniform DAH sandwich models with large NPRs generally yield better sound insulation behaviors. Moreover, the considered DAH sandwich models insulate less sound but are far stiffer than the conventional hexagonal honeycomb sandwich models.

(3) The programmable optimized gradient INPR models yield higher STL values than the optimized DNPR models, with improvements of $6.52 \mathrm{~dB}$ and $2.52 \mathrm{~dB}$ for the specified frequency cases and a large bending stiffness; however, a lower overall sound insulation was observed. For the gradient sandwich models, the DAHs with large NPRs in the upper layers improve 


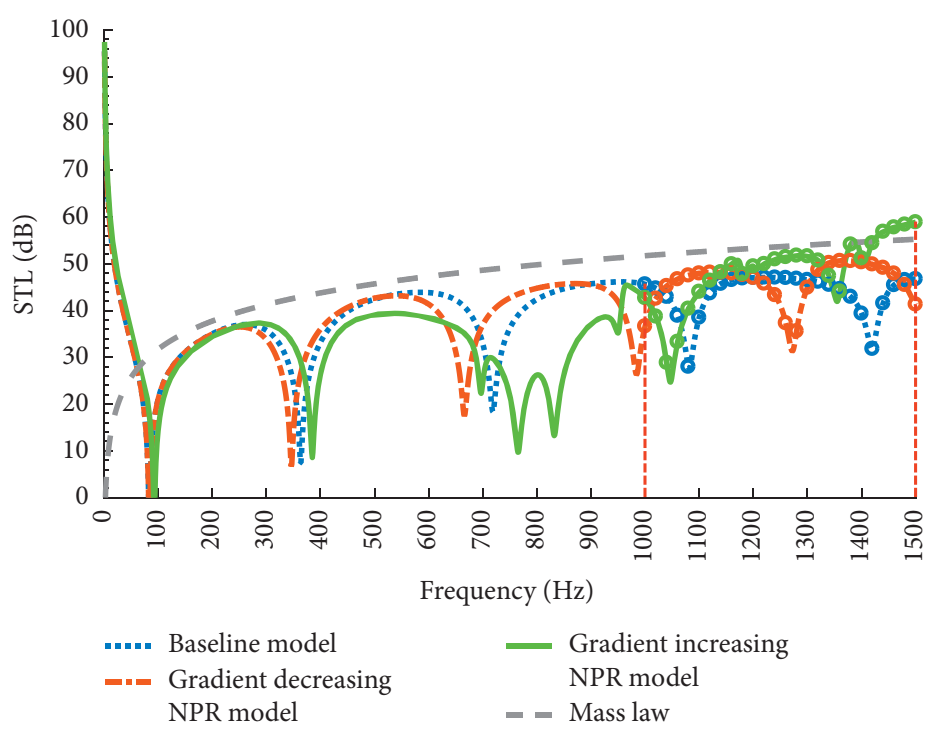

(a)

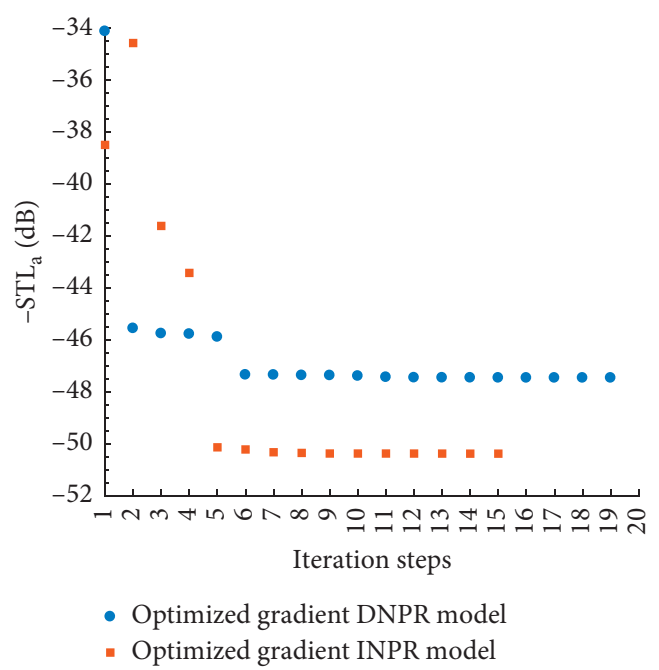

(b)

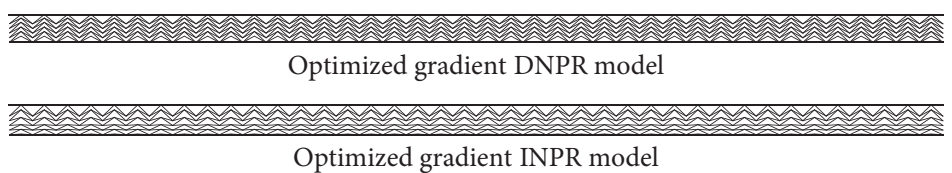

(c)
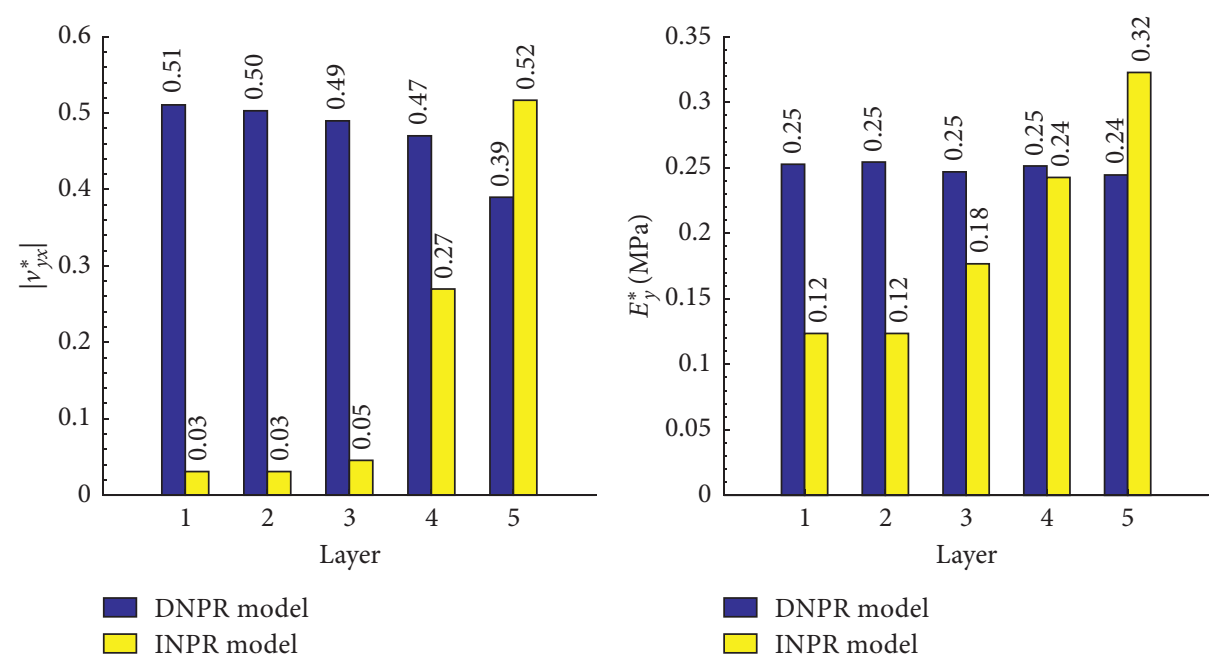

(d)

Figure 16: Optimal design from 1000 to $1500 \mathrm{~Hz}$ : (a) STLs of the baseline and optimized models; (b) iteration steps; (c) configurations of the optimized gradient NPR models; (d) mechanical properties of the DAHs within the optimized gradient NPR models.

Table 3: Characteristic parameters of the baseline and optimized models for optimal design from 1000 to $1500 \mathrm{~Hz}$.

\begin{tabular}{lcccc}
\hline Model & $\Theta_{2}(\mathrm{deg})$ & $\mathrm{STL}_{\mathrm{a}}(\mathrm{dB})$ & $f_{0}(\mathrm{~Hz})$ & $\mathrm{STL}_{\mathrm{o}}(\mathrm{dB})$ \\
\hline Baseline & {$[55.00,55.00,55.00,55.00,55.00]$} & 45.36 & 83.72 & 39.67 \\
Optimized gradient DNPR & {$[46.71,47.14,47.14,48.31,51.53]$} & 47.87 & 83.73 & 40.16 \\
Optimized gradient INPR & {$[65.00,65.00,55.51,46.78,46.25]$} & 50.39 & 92.91 & 38.31 \\
\hline
\end{tabular}

the potential for obtaining an optimized target STL, and the DAHs in the bottom layers affect mainly the STL curve shapes and govern the overall noise insulation properties.
(4) Further consideration could be given to the vibroacoustic performance and design of the proposed structures at high frequencies and under other excitation conditions. Furthermore, the design 
variables can also be extended to consider more than a single feature angle. Moreover, further studies could be conducted to investigate gradient auxetic metamaterials with varying topologies.

\section{Data Availability}

The programming data used to support the findings of this study are available from the corresponding author upon request.

\section{Conflicts of Interest}

The authors declare that they have no conflicts of interest.

\section{Acknowledgments}

The support for this work provided by the National Natural Science Foundation of China (51479115), High-Tech Ship Research Projects by MIIT ([2014]148 and [2016]548), and Opening Project by the State Key Laboratory of Ocean Engineering (GKZD010071) is gratefully acknowledged.

\section{References}

[1] C. Huang and L. Chen, "Negative Poisson's ratio in modern functional materials," Advanced Materials, vol. 28, no. 37, pp. 8079-8096, 2016.

[2] K. E. Evans and A. Alderson, "Auxetic materials: functional materials and structures from lateral thinking!," Advanced Materials, vol. 12, no. 9, pp. 617-628, 2000.

[3] Y. Prawoto, "Seeing auxetic materials from the mechanics point of view: a structural review on the negative Poisson's ratio," Computational Materials Science, vol. 58, pp. 140-153, 2012.

[4] H. M. A. Kolken and A. A. Zadpoor, "Auxetic mechanical metamaterials," RSC Advances, vol. 7, no. 9, pp. 5111-5129, 2017.

[5] R. Lakes, "Foam structures with a negative Poisson's ratio," Science, vol. 235, no. 4792, pp. 1038-1040, 1987.

[6] L. J. Gibson and M. F. Ashby, Cellular Solids: Structure and Properties, Cambridge University, Cambridge, UK, 1999.

[7] Q. Zhang, X. Yang, P. Li et al., "Bioinspired engineering of honeycomb structure - using nature to inspire human innovation," Progress in Materials Science, vol. 74, pp. 332-400, 2015.

[8] K. E. Evans, B. D. Caddock, and M. J. Nobes, "Microporous materials with negative Poisson's ratios. II. Mechanisms and interpretation," Journal of Physics D: Applied Physics, vol. 22, no. 12, pp. 1883-1887, 1989.

[9] S. Babaee, J. Shim, J. C. Weaver, E. R. Chen, N. Patel, and K. Bertoldi, "3D soft metamaterials with negative Poisson's ratio," Advanced Materials, vol. 25, no. 36, pp. 5044-5049, 2013.

[10] R. Lakes, “Deformation mechanisms in negative Poisson's ratio materials: structural aspects," Journal of Materials Science, vol. 26, no. 9, pp. 2287-2292, 1991.

[11] J. N. Grima and K. E. Evans, "Auxetic behavior from rotating squares,” Journal of Materials Science Letters, vol. 19, no. 17, pp. 1563-1565, 2000.

[12] V. D’Alessandro, G. Petrone, F. Franco, and S. De Rosa, “A review of the vibroacoustics of sandwich panels: models and experiments," Journal of Sandwich Structures and Materials, vol. 15, no. 5, pp. 541-582, 2013.
[13] J. Wu, "Topology optimization studies for light weight acoustic panels," M.S. thesis, University of Toronto, Toronto, Canada, 2016.

[14] J. A. Moore and R. H. Lyon, "Sound transmission loss characteristics of sandwich panel constructions," Journal of the Acoustical Society of America, vol. 89, no. 2, pp. 777-791, 1991.

[15] P. Thamburaj and J. Q. Sun, "Optimization of anisotropic sandwich beams for higher sound transmission loss," Journal of Sound and Vibration, vol. 254, no. 1, pp. 23-36, 2002.

[16] M. El-Raheb and P. Wagner, "Transmission of sound across a trusslike periodic panel; 2-D analysis," Journal of the Acoustical Society of America, vol. 102, no. 4, pp. 2176-2183, 1997.

[17] M. Ruzzene, "Vibration and sound radiation of sandwich beams with honeycomb truss core," Journal of Sound and Vibration, vol. 277, no. 4-5, pp. 741-763, 2004.

[18] D. Griese, J. D. Summers, and L. Thompson, "The effect of honeycomb core geometry on the sound transmission performance of sandwich panels," Journal of Vibration and Acoustics, vol. 137, no. 2, article 021011, 2014.

[19] A. Spadoni and M. Ruzzene, "Structural and acoustic behavior of chiral truss-core beams," Journal of Vibration and Acoustics, vol. 128, no. 5, pp. 616-626, 2006.

[20] H. Denli and J. Q. Sun, "Structural-acoustic optimization of sandwich structures with cellular cores for minimum sound radiation," Journal of Sound and Vibration, vol. 301, no. 1-2, pp. 93-105, 2007.

[21] F. Franco, K. A. Cunefare, and M. Ruzzene, "Structuralacoustic optimization of sandwich panels," Journal of Vibration and Acoustics, vol. 129, no. 3, pp. 330-340, 2007.

[22] R. Galgalikar and L. L. Thompson, "Design optimization of honeycomb core sandwich panels for maximum sound transmission loss," Journal of Vibration and Acoustics, vol. 138, no. 5, article 051005, 2016.

[23] Q. Li and D. Yang, "Mechanical and acoustic performance of sandwich panels with hybrid cellular cores," Journal of Vibration and Acoustics, vol. 140, no. 6, article 061016, 2018.

[24] A. S. Phani, J. Woodhouse, and N. A. Fleck, "Wave propagation in two-dimensional periodic lattices," Journal of the Acoustical Society of America, vol. 119, no. 4, pp. 1995-2005, 2016.

[25] S. Ehsan Moosavimehr and A. Srikantha Phani, "Sound transmission loss characteristics of sandwich panels with a truss lattice core," Journal of the Acoustical Society of America, vol. 141, no. 4, pp. 2921-2932, 2017.

[26] T. J. Cui, M. Q. Qi, X. Wan, J. Zhao, and Q. Cheng, "Coding metamaterials, digital metamaterials and programmable metamaterials," Light: Science and Applications, vol. 3, no. 10, p. e218, 2014.

[27] T. C. Lim, "Functionally graded beam for attaining Poissoncurving," Journal of Materials Science Letters, vol. 21, no. 24, pp. 1899-1901, 2002.

[28] Y. Han and W. Lu, "Evolutionary design of nonuniform cellular structures with optimized Poisson's ratio distribution," Materials and Design, vol. 141, pp. 384-394, 2018.

[29] Y. Hou, Y. H. Tai, C. Lira, F. Scarpa, J. R. Yates, and B. Gu, "The bending and failure of sandwich structures with auxetic gradient cellular cores," Composites Part A: Applied Science and Manufacturing, vol. 49, pp. 119-131, 2013.

[30] Y. Hou, R. Neville, F. Scarpa, C. Remillat, B. Gu, and M. Ruzzene, "Graded conventional-auxetic Kirigami sandwich structures: flatwise compression and edgewise loading," Composites Part B: Engineering, vol. 59, pp. 3342, 2014. 
[31] C. Yang, H. D. Vora, and Y. Chang, "Behavior of auxetic structures under compression and impact forces," Smart Materials and Structures, vol. 27, no. 2, article 025012, 2018.

[32] C. Lira, F. Scarpa, and R. Rajasekaran, "A gradient cellular core for aeroengine fan blades based on auxetic configurations," Journal of Intelligent Material Systems and Structures, vol. 22, no. 9, pp. 907-917, 2011.

[33] L. Boldrin, S. Hummel, F. Scarpa et al., "Dynamic behaviour of auxetic gradient composite hexagonal honeycombs," Composite Structures, vol. 149, pp. 114-124, 2016.

[34] M. S. Mazloomi, M. Ranjbar, L. Boldrin, F. Scarpa, S. Patsias, and N. Ozada, "Vibroacoustics of 2D gradient auxetic hexagonal honeycomb sandwich panels," Composite Structures, vol. 187, pp. 593-603, 2018.

[35] J. X. Qiao and C. Q. Chen, "Impact resistance of uniform and functionally graded auxetic double arrowhead honeycombs," International Journal of Impact Engineering, vol. 83, pp. 47-58, 2015.

[36] T.-C. Lim, "A 3D auxetic material based on intersecting double arrowheads," Physica Status Solidi (b), vol. 253, no. 7, pp. 1252-1260, 2016.

[37] N. Novak, M. Vesenjak, and Z. Ren, "Computational simulation and optimization of functionally graded auxetic structures made from inverted tetrapods," Physica Status Solidi (b), vol. 254, no. 12, p. 1600753, 2017.

[38] W. Hou, X. Yang, W. Zhang, and Y. Xia, "Design of energydissipating structure with functionally graded auxetic cellular material," International Journal of Crashworthiness, vol. 23, no. 4, pp. 366-376, 2017.

[39] U. Lee, Spectral Element Method in Structural Dynamics, John Wiley \& Sons, New York, USA, 2009.

[40] H. Yang, H. Li, and H. Zheng, "A structural-acoustic optimization of two-dimensional sandwich plates with corrugated cores," Journal of Vibration and Control, vol. 23, no. 18, pp. 3007-3022, 2017.

[41] W. H. Wittrick and F. W. Williams, "A general algorithm for computing natural frequencies of elastic structures," The Quarterly Journal of Mechanics and Applied Mathematics, vol. 24, no. 3, pp. 263-284, 1971.

[42] F. W. Williams, "An algorithm for exact eigenvalue calculations for rotationally periodic structures," International Journal for Numerical Methods in Engineering, vol. 23, no. 4, pp. 609-622, 1986.

[43] S. Yuan, K. Ye, and F. W. Williams, "Second order modefinding method in dynamic stiffness matrix methods," Journal of Sound and Vibration, vol. 269, no. 3-5, pp. 689-708, 2004.

[44] L. Cheng, Y. Y. Li, and J. X. Gao, "Energy transmission in a mechanically-linked double-wall structure coupled to an acoustic enclosure," Journal of the Acoustical Society of America, vol. 117, no. 5, pp. 2742-2751, 2005.

[45] E. G. Williams, Fourier acoustics: Sound radiation and Nearfield Acoustical Holography, Academic, London, UK, 1999. 


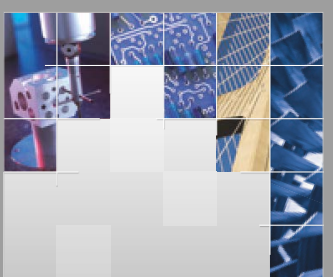

\section{Enfincering}
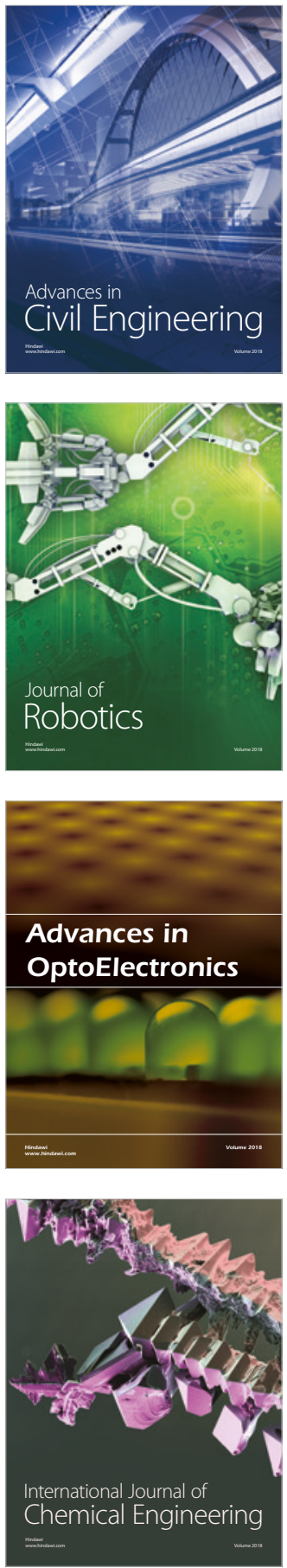

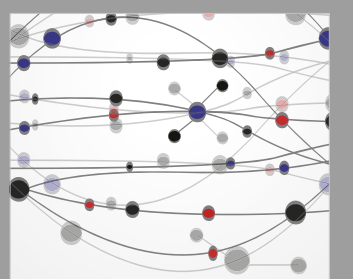

\section{Rotating \\ Machinery}

The Scientific World Journal

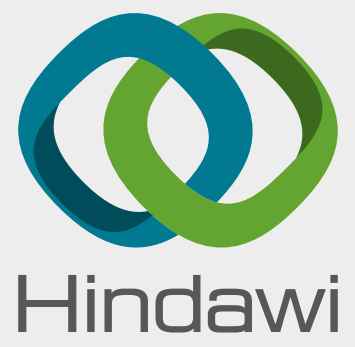

Submit your manuscripts at

www.hindawi.com
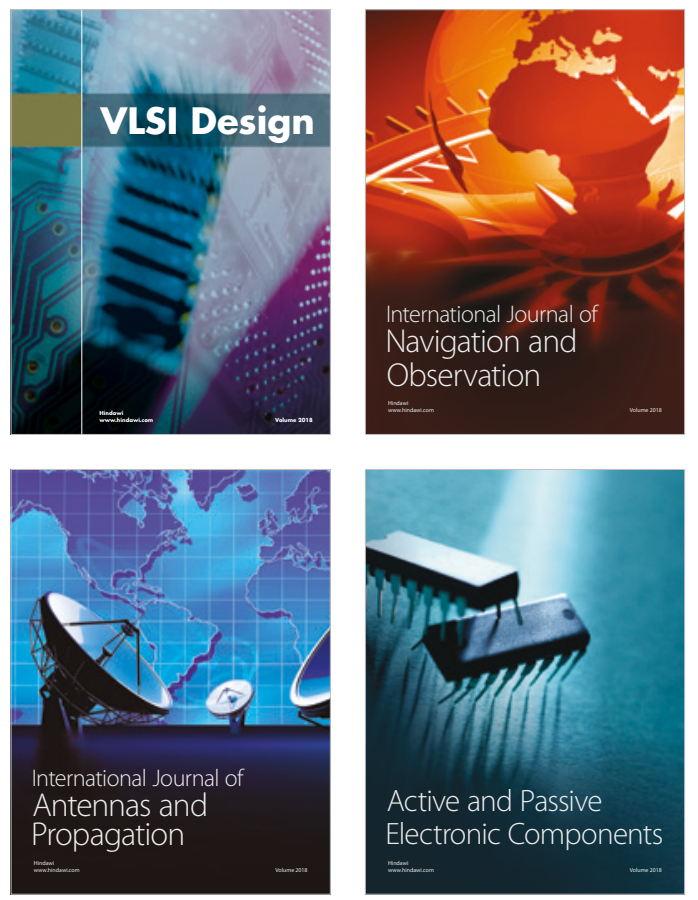
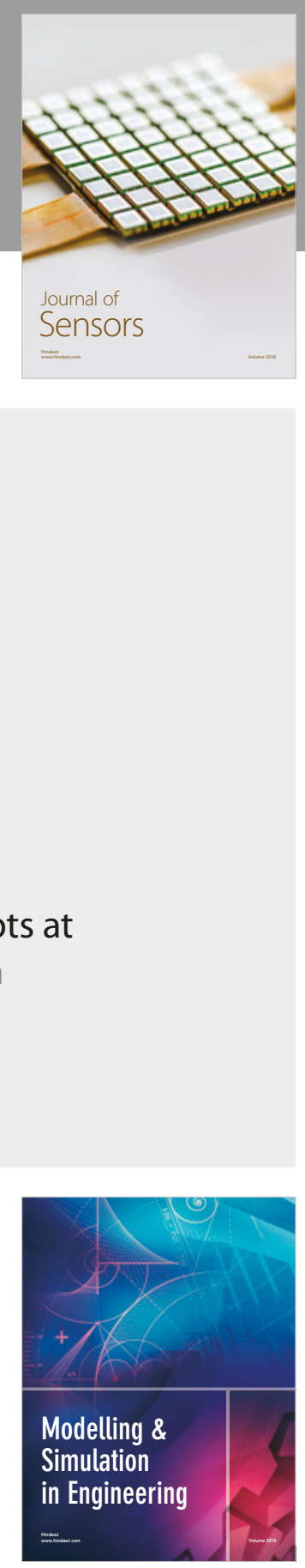

\section{Advances \\ Multimedia}
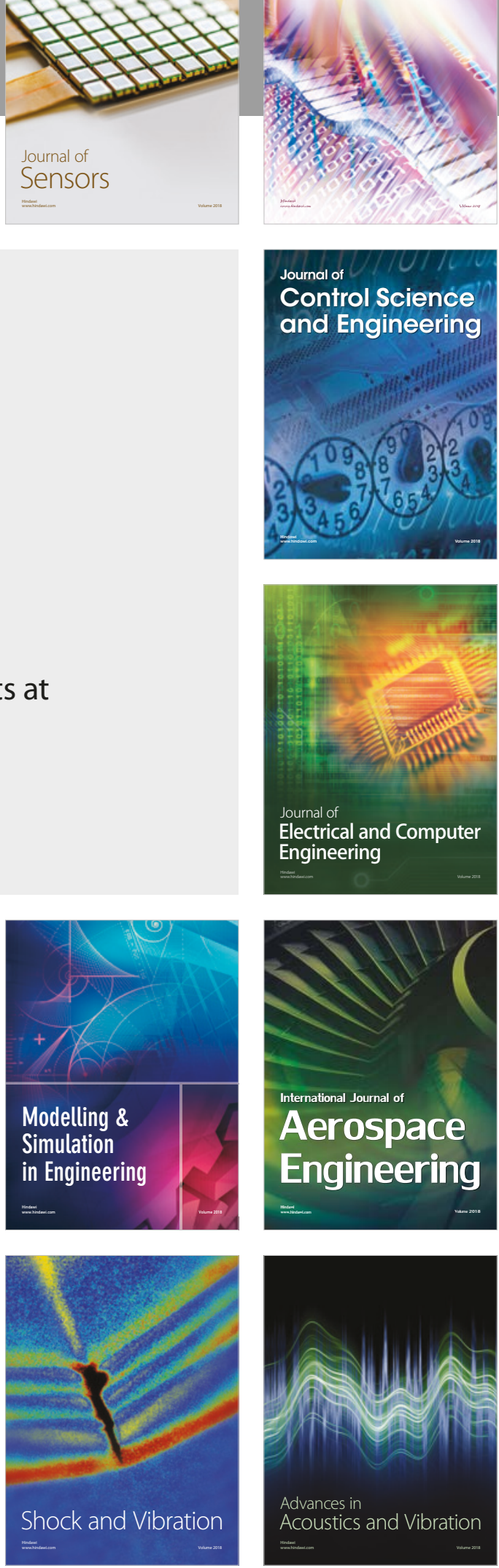